Prepared in cooperation with the Colorado River Water Conservation District, Eagle County, Eagle River Water and Sanitation District, Upper Eagle Regional Water Authority, Colorado Department of Transportation, City of Aurora, Town of Eagle, Town of Gypsum, Town of Minturn, Town of Vail, Vail Resorts, Colorado Springs Utilities, Denver Water, and the U.S. Department of Agriculture Forest Service

\title{
Macroinvertebrate-Based Assessment of Biological Condition at Selected Sites in the Eagle River Watershed, Colorado, 2000-07
}

Scientific Investigations Report 2010-5148 
Cover: Example of mixed site class used to evaluate the effect of land cover on biological condition in the Eagle River watershed, Colorado, 2000-07. 


\section{Macroinvertebrate-Based Assessment of Biological Condition at Selected Sites in the Eagle River Watershed, Colorado, 2000-07}

By Robert E. Zuellig, James F. Bruce, Brian D. Healy, and Cory A. Williams

Prepared in cooperation with the Colorado River Water Conservation District, Eagle County, Eagle River Water and Sanitation District, Upper Eagle Regional Water Authority, Colorado Department of Transportation, City of Aurora, Town of Eagle, Town of Gypsum, Town of Minturn, Town of Vail, Vail Resorts, Colorado Springs Utilities, Denver Water, and the U.S. Department of Agriculture Forest Service

Scientific Investigations Report 2010-5148 


\title{
U.S. Department of the Interior \\ KEN SALAZAR, Secretary \\ U.S. Geological Survey \\ Marcia K. McNutt, Director
}

\section{U.S. Geological Survey, Reston, Virginia: 2010}

\author{
For more information on the USGS — the Federal source for science about the Earth, its natural and living resources, \\ natural hazards, and the environment, visit http://www.usgs.gov or call 1-888-ASK-USGS \\ For an overview of USGS information products, including maps, imagery, and publications, \\ visit http://www.usgs.gov/pubprod \\ To order this and other USGS information products, visit http://store.usgs.gov
}

Any use of trade, product, or firm names is for descriptive purposes only and does not imply endorsement by the U.S. Government.

Although this report is in the public domain, permission must be secured from the individual copyright owners to reproduce any copyrighted materials contained within this report.

Suggested citation:

Zuellig, R.E., Bruce, J.F., Healy, B.D., Williams, C.A., 2010, Macroinvertebrate-based assessment of biological condition at selected sites in the Eagle River watershed, Colorado, 2000-07: U.S. Geological Survey Scientific Investigations Report 2010-5148, 19 p. 


\section{Contents}

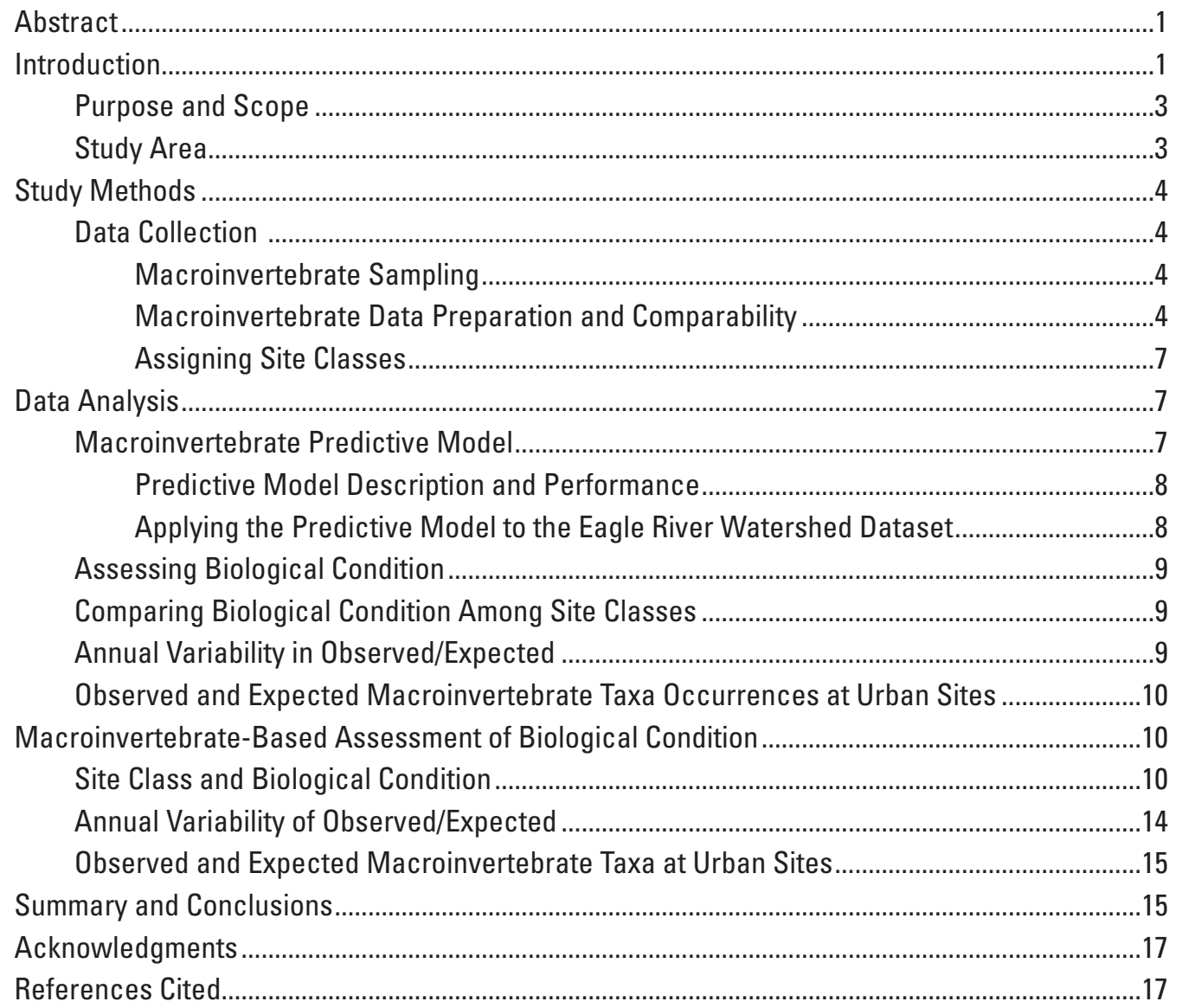

\section{Figures}

1. Map showing land cover and selected streams and cities in the Eagle River watershed, Colorado, 2000-07

2. Map showing selected sites and Eagle River watershed subregions, Colorado, 2000-07.

3. Representative photographs of the four site classes used to evaluate the effect of land cover on biological condition in the Eagle River watershed, Colorado, 2000-07.

4. Graph showing summary of observed/expected values calculated from 124 macroinvertebrate samples collected at 73 sites grouped by different subregions of the Eagle River watershed, Colorado, 2000-07

5. Graph showing distribution of macroinvertebrate observed/expected values among undeveoped, adjacent road or highway or both, mixed, and urban site classes.

6. Map showing site location, site class, biological condition, and Eagle River watershed subregions, Colorado 
7. Graph showing distribution of observed/expected values collected from 14 sites $(6$ undeveloped sites, 20 samples; 4 Black Gore Creek sites,

17 samples; 4 Gore Creek sites, 18 samples) representing 3 to 5 years of annual variability between 2000 and 2007

8. Graph showing frequencies of observed $\left(\mathrm{O}_{\mathrm{f}}\right)$ and expected $\left(\mathrm{E}_{\mathrm{f}}\right)$ occcurrence of 19 invertebrate taxa that statistically occurred more or less frequently than predicted or were impartial to conditions at urban sites in the Eagle River watershed, Colorado, 2000-07

\section{Tables}

1. Description of sites, data source, sample types, and biological assessments of selected sites in the Eagle River watershed, Colorado, 2000-07.

2. Site and data description where 3 to 5 years of annual macroinvertebrate samples were collected in the Eagle River watershed, Colorado, 2000-07 


\section{Conversion Factors, Datum, and Abbreviations}

Inch/Pound to SI

\begin{tabular}{|c|c|c|}
\hline Multiply & By & To obtain \\
\hline \multicolumn{3}{|c|}{ Length } \\
\hline foot $(\mathrm{ft})$ & 0.3048 & meter $(\mathrm{m})$ \\
\hline meters (m) & 3.2808 & feet $(\mathrm{ft})$ \\
\hline mile (mi) & 1.609 & kilometer (km) \\
\hline \multicolumn{3}{|c|}{ Area } \\
\hline acre & 0.004047 & square kilometer $\left(\mathrm{km}^{2}\right)$ \\
\hline square foot $\left(\mathrm{ft}^{2}\right)$ & 929.0 & square centimeter $\left(\mathrm{cm}^{2}\right)$ \\
\hline square foot $\left(\mathrm{ft}^{2}\right)$ & 0.09290 & square meter $\left(\mathrm{m}^{2}\right)$ \\
\hline square mile $\left(\mathrm{mi}^{2}\right)$ & 259.0 & hectare (ha) \\
\hline square mile $\left(\mathrm{mi}^{2}\right)$ & 2.590 & square kilometer $\left(\mathrm{km}^{2}\right)$ \\
\hline
\end{tabular}

Temperature in degrees Celsius $\left({ }^{\circ} \mathrm{C}\right)$ may be converted to degrees Fahrenheit $\left({ }^{\circ} \mathrm{F}\right)$ as follows:

$$
{ }^{\circ} \mathrm{F}=\left(1.8 \mathrm{x}^{\circ} \mathrm{C}\right)+32
$$

Temperature in degrees Fahrenheit $\left({ }^{\circ} \mathrm{F}\right)$ may be converted to degrees Celsius $\left({ }^{\circ} \mathrm{C}\right)$ as follows:

$$
{ }^{\circ} \mathrm{C}=\left({ }^{\circ} \mathrm{F}-32\right) / 1.8
$$

Vertical coordinate information is referenced to the North American Vertical Datum of 1988 (NAVD 88).

Horizontal coordinate information is referenced to the North American Datum of 1983 (NAD 83).

Elevation, as used in this report, refers to distance above the vertical datum. 


\title{
Abbreviations
}

\author{
ANOVA Analysis of variance \\ ARH Adjacent road or highway or both classified site \\ CDLG Colorado Department of Local Governments \\ CDOT Colorado Department of Transportation \\ CDPHE Colorado Department of Public Health and Environment \\ ERW Eagle River Watershed \\ GIS Geographical Information System \\ NAWQA National Water-Quality Assessment Program \\ 0/E Ratio of observed (0) taxa to expected (E) taxa \\ OTU Operational taxonomic unit \\ PRISM Parameter-elevation Regressions on Independent Slopes Model \\ SD Standard Deviation \\ USEPA U.S. Environmental Protection Agency \\ USGS U.S. Geological Survey \\ FS U.S. Department of Agriculture Forest Service
}




\title{
Macroinvertebrate-Based Assessment of Biological Condition at Selected Sites in the Eagle River Watershed, Colorado, 2000-07
}

\author{
By Robert E. Zuellig, James F. Bruce, Brian D. Healy, and Cory A. Williams
}

\section{Abstract}

The U.S. Geological Survey (USGS), in cooperation with the Colorado River Water Conservation District, Eagle County, Eagle River Water and Sanitation District, Upper Eagle Regional Water Authority, Colorado Department of Transportation, City of Aurora, Town of Eagle, Town of Gypsum, Town of Minturn, Town of Vail, Vail Resorts, Colorado Springs Utilities, Denver Water, and the U.S. Department of Agriculture Forest Service (FS), compiled macroinvertebrate ( 73 sites, 124 samples) data previously collected in the Eagle River watershed from selected USGS and FS studies, 2000-07. These data were analyzed to assess the biological condition (that is, biologically "degraded" or "good") at selected sites in the Eagle River watershed and determine if site class (for example, urban or undeveloped) described biological condition.

An independently developed predictive model was applied to calculate a site-specific measure of taxonomic completeness for macroinvertebrate communities, where taxonomic completeness was expressed as the ratio of observed (O) taxa to those expected (E) to occur at each site. Macroinvertebrate communities were considered degraded at sites were $\mathrm{O} / \mathrm{E}$ values were less than 0.80 , indicating that at least 20 percent of expected taxa were not observed. Sites were classified into one of four classes (undeveloped, adjacent road or highway or both, mixed, urban) using a combination of riparian land-cover characteristics, examination of topographic maps and aerial imagery, screening for exceedances in waterquality standards, and best professional judgment. Analysis of variance was used to determine if site class accounted for variability in mean macroinvertebrate $\mathrm{O} / \mathrm{E}$ values. Finally, macroinvertebrate taxa observed more or less frequently than expected at urban sites were identified.

This study represents the first standardized assessment of biological condition of selected sites distributed across the Eagle River watershed. Of the 73 sites evaluated, just over half (55 percent) were considered in good biological condition $(\mathrm{O} / \mathrm{E}$ greater than 0.80$)$. The remaining sites were either consistently biologically degraded ( 30 percent; O/E less than
$0.80)$ or varied annually between good and degraded condition (15 percent; O/E is less than or greater than 0.80 ). Sites primarily affected by urbanization were among the most severely degraded (lowest $\mathrm{O} / \mathrm{E}$ values) when compared to other site classes. Although most urban sites were among the most severely degraded (lowest $\mathrm{O} / \mathrm{E}$ values), a few sites had nearly intact macroinvertebrate communities (O/E near 1.0). Similar observations were noted among sites classified as mixed.

Thirteen macroinvertebrate taxa were identified that occurred more or less frequently than expected at urban sites. Additionally, six other taxa were impartial (tolerant) to the same conditions. Combined, these 19 taxa provide an opportunity to enhance the interpretation of future studies in the Eagle River watershed, but will require better insight into the responses of these taxa to specific stressors. Understanding the sources of variability affecting biological condition along with why some sites expected to be degraded, but showed otherwise, will have clear implications for mitigation efforts. Integrating results of this study with field and laboratory investigations will greatly enhance the ability to identify causal factors affecting biological condition at degraded sites, the logical next step. Information generated from such integrative studies will be imperative for well targeted mitigation efforts in the Eagle River watershed.

\section{Introduction}

Eagle River, a tributary of the Colorado River, drains an area of approximately 970 square miles $\left(\mathrm{mi}^{2}\right)$ in Colorado flowing from the Continental Divide through Red Cliff, Minturn, Avon, Edwards, Wolcott, Eagle, and Gypsum (fig. 1), and continues downstream approximately 6 miles to the confluence with the Colorado River at Dotsero. Several environmental factors that affect the biological condition of streams have been modified to some degree in the Eagle River watershed (for example, surface-water hydrology, water quality, land cover, and riparian area disturbance), where changes are expected to intensify in the future (Bledsoe and others, 2005). State and local agencies are concerned about the effects 


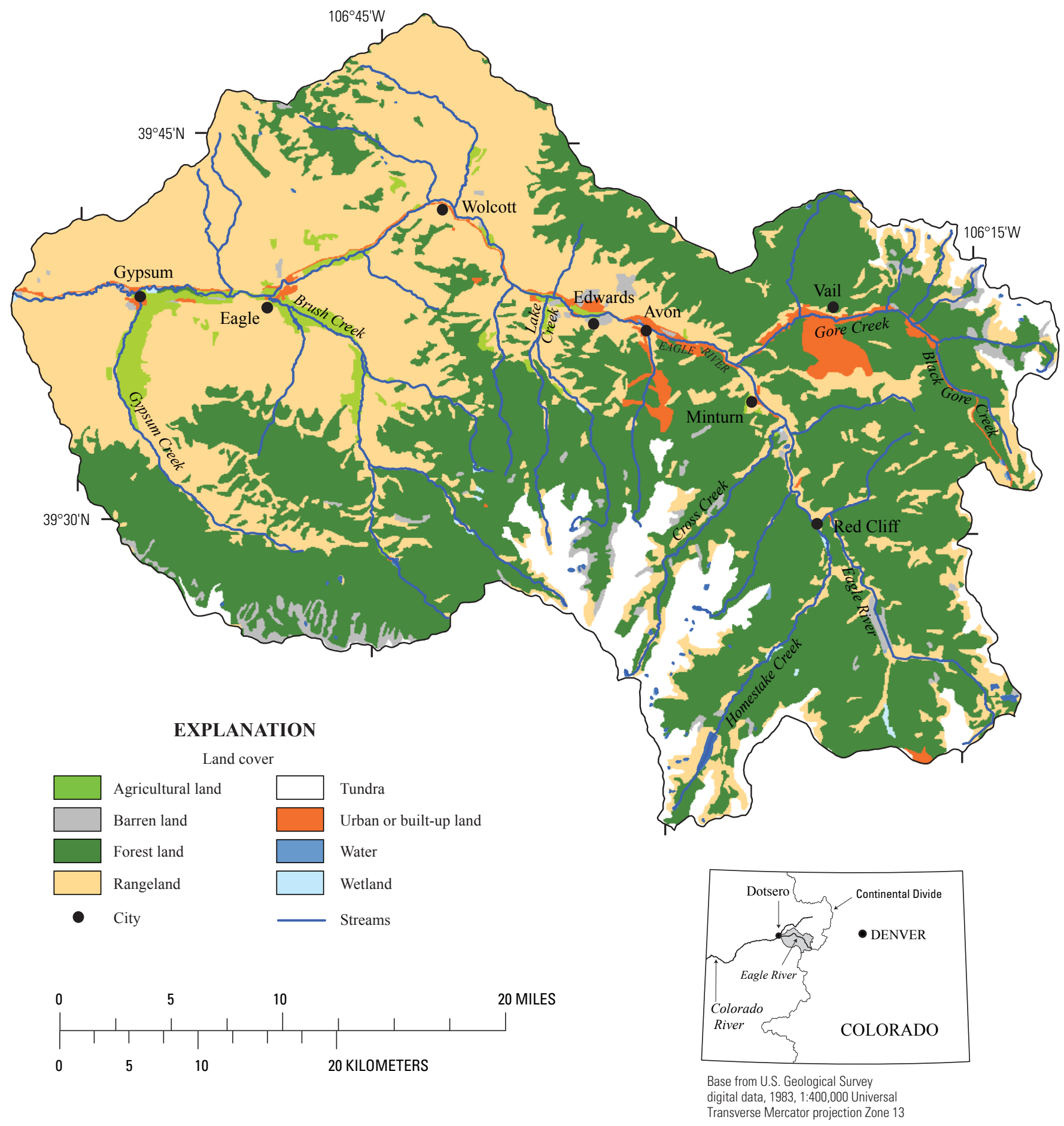

Figure 1. Land cover and selected streams and cities in the Eagle River watershed, Colorado, 2000-07. 
of increasing urban development and human population on water quality and the biological condition of regional streams (Deacon and Spahr, 1998). Current (2010) and expected future development along the Eagle River and Gore Creek corridors could adversely affect water quality and stream biota (Bledsoe and others, 2005; Wynn and others, 2001).

Several local studies have noted site-specific differences in community structure of fish, macroinvertebrate, and diatom communities in the upper part of the Eagle River watershed (Wynn and others, 2001; Hydrosphere, 2007; Healy, 2008), but relatively few have made clear connections between observed patterns and specific stressors (Vieira and others, 2005). Even less is known about factors affecting the biological condition downstream from Avon to the Colorado River.

Local entities are interested in better understanding how specific land-management activities affect the biological and water quality of the Eagle River watershed in order to guide watershed-wide water-resource management. In response to these needs, the U.S. Geological Survey (USGS), in cooperation with the Colorado River Water Conservation District, Eagle County, Eagle River Water and Sanitation District, Upper Eagle Regional Water Authority, Colorado Department of Transportation, City of Aurora, Town of Eagle, Town of Gypsum, Town of Minturn, Town of Vail, Vail Resorts, Colorado Springs Utilities, Denver Water, and the U.S. Department of Agriculture Forest Service (FS), compiled previously collected macroinvertebrate data from the Eagle River watershed. These data were analyzed to assess the biological condition (that is, biologically "degraded" or "good") of selected sites. This assessment of biological condition herein differs from what has been done locally in the past because regionally derived biological assessment tools are now available, which facilitates quantitative predictions of the loss of biological diversity in Eagle River watershed streams based on regional reference conditions. This study represents the first standardized assessment of biological condition of selected sites within the Eagle River watershed.

\section{Purpose and Scope}

This report presents a macroinvertebrate-based assessment of biological condition using a compilation of macroinvertebrate data collected by USGS and FS from up to 73 sites in the Eagle River watershed between 2000 and 2007. Data were compiled from independent studies; therefore, all data types were not collected in all years at all sites. The objectives of this report were to (1) assess the biological condition of selected sites in the Eagle River watershed using macroinvertebrate communities and (2) evaluate whether the dominant types of land cover were associated with biological condition. Additionally, short-term annual variability ( 3 to 5 years) in observed taxa to those expected $(\mathrm{O} / \mathrm{E})$ values was described and macroinvertebrate taxa that occurred more or less frequently than predicted at urban sites were identified. All macroinvertebrate data evaluated in this report, as well as some accompanying diatom data not presented in this report, are documented in Zuellig and Bruce, (2010), available online at $h t t p: / / p u b s . u s g s . g o v / d s / 5021$.

\section{Study Area}

Several previous studies provide a detailed description of the Eagle River watershed study area (for example, Bledsoe and others, 2005). In brief, the Eagle River watershed (ERW) drains approximately $950 \mathrm{mi}^{2}$ where the river flows north from its headwaters to the confluence of Gore Creek near Avon, then generally west to its terminus where it joins the Colorado River at Dotsero. Major tributaries include Gore Creek, Homestake Creek, Cross Creek, Lake Creek, and Brush Creek (fig. 1). Gore Creek, the largest tributary in the upper part of the watershed, flows through Vail draining approximately $102 \mathrm{mi}^{2}$ before it reaches its confluence with the Eagle River near Minturn. Land-surface elevations range from approximately 14,005 to $6,130 \mathrm{ft}$.

The geology of the ERW is highly variable, which differentially affects water-quality conditions in discrete parts of the watershed. For example, a few tributaries near Wolcott drain highly erosive soils (for example, soils developed within Pierre Shale) that contribute fine sediments to the Eagle River during snowmelt or summertime rainfall events (Bledsoe and others, 2005). Additionally, the southern part of the watershed (roughly the area upstream from Minturn, fig. 1) is part of the Colorado Mineral Belt (Wilson and Sims, 2003), which has contributed to a multibillion dollar mining industry in Colorado (Tweto, 1979).

Temperature, precipitation, and streamflow patterns are typical of regional river watersheds in Colorado. Monthly average temperatures typically range from $14^{\circ} \mathrm{F}$ in January to $56^{\circ} \mathrm{F}$ in July in the Vail area and from $18^{\circ} \mathrm{F}$ to $67^{\circ} \mathrm{F}$ near Eagle. Precipitation typically ranges from 22 inches per year in the higher elevations to around 11 inches in the lower valleys (National Oceanic and Atmospheric Administration, 2009). Much of the precipitation falls in the form of snow throughout the winter months resulting in a snowmelt-driven hydrograph (Bledsoe and others, 2005). Typically, streamflow increases in early spring as snow melts at lower elevations, peaks around mid June, and slowly recedes throughout the summer months. During late spring through late summer, streamflow is supplemented by a combination of localized high-intensity convective thunderstorms. Base flow is sustained by tributary groundwater when thunderstorms diminish in late summer until the snow begins to melt the following spring. Streamflow is intensively managed throughout the watershed by storing and diverting water for municipal and agricultural use, maintaining minimum instream flow requirements, and snow making for local ski areas.

In 2001, approximately 5 percent of the ERW was considered developed (urban or agricultural) and 95 percent was undeveloped (primarily forested) (U.S. Geological Survey, 2007). Most developed lands in the watershed generally occur 
along stream corridors and can account for as much as 35 percent of land cover of stream-side areas (Bledsoe and others, 2005). Ski tourism and second home development currently are the largest contributors to increases in developed lands in the ERW. Human population in the area is projected to nearly double by 2030 (Colorado Department of Local Government, 2003), which is expected to increase developed lands along stream corridors (Bledsoe and others, 2005).

\section{Study Methods}

One-hundred twenty-four macroinvertebrate samples collected from 73 sites (fig. 2) were compiled from various USGS and FS projects in the ERW between 2000 and 2007 (table 1). Data were compiled from various projects and agencies; therefore, not all data types were collected at all sites with the same frequency. For example, some sites were visited only once; whereas, others were visited annually during a 5-year period. Therefore by necessity, various combinations of samples and sites were used to address each objective. Site assessments of biological condition were made by applying an independently developed indicator and establishing a threshold value defining biological degradation.

\section{Data Collection}

\section{Macroinvertebrate Sampling}

The USGS collected semiquantitative-macroinvertebrate samples during 2000 and 2001 following National WaterQuality Assessment (NAWQA) Program protocols described by Cuffney and others (1993). In general, five discrete collections were made from riffle areas at each site with a slack sampler equipped with 500-micron mesh and a $2.69 \mathrm{ft}^{2}$ $\left(0.25 \mathrm{~m}^{2}\right)$ sampling grid along a pre-defined stream reach $(20$ times the stream width, Fitzpatrick and others, 1998). The contents of the five collections were composited, elutriated, and poured through a 500-micron mesh sieve, and preserved with 10-percent buffered formalin in the field. All samples were transported to Chadwick and Associates in Littleton, Colo., where they were processed using methods described by Klemm and others (1990). All taxa were identified to the lowest practical resolution (genus or species) and enumerated. All USGS macroinvertebrate samples were collected during base-flow conditions during September; some USGS sites were sampled in both 2000 and 2001 (table 1).

The FS collected macroinvertebrate samples between 2003 and 2007 using methods described by Hawkins and others (2001) (table 1). In general, eight macroinvertebrate samples were collected and composited from four riffle locations within an 8-ft stream reach (length of stream encompassing four distinct riffle areas) using a surber sampler equipped with 500-micron mesh and a 1- $\mathrm{ft}^{2}$ sampling grid. Macroinvertebrate samples from each site were composited and preserved in the field and sent to Aquatics Associates, Inc., Fort Collins, Colo., for sample processing following protocols developed by Klemm and others (1990). Additionally, the FS sampled macroinvertebrate communities from a series of sites along the Eagle River in the upper part of the watershed (table 1, fig. 2, sites 5, 9, 11-12, 17, 22, and 24). At these sites, five replicate macroinvertebrate samples were collected from a single riffle using a Hess sampler (each replicate represents $0.923 \mathrm{ft}^{2}$ of streambed) equipped with 500-micron mesh and preserved separately in the field and sent to Aquatics Associates Inc., Ft. Collins, Colo. for taxa identification and sample processing (Klemm and others, 1990). All taxa in each sample were identified to the lowest practical resolution (for example, genus or species) and enumerated. All FS macroinvertebrate samples were collected during base-flow conditions during September.

\section{Macroinvertebrate Data Preparation and Comparability}

Three adjustments were made to the compiled ERW data in preparation for data analysis. First, taxonomic names used by the different laboratories were harmonized. Second, the laboratory results were composited from the five FS Hess samples (Cuffney and others, 1993; Hawkins and others, 2001). Third, the raw data counts were standardized for each sample to 300 by randomly resampling individuals without replacement using a personal computer. Such adjustments to raw data counts can help alleviate the effect of varying levels of sampling effort (for example, area sampled in the field or subsample target counts) on estimates of taxa richness (Peterson and Zumberge, 2006), even when different sampling devices are used (Cao and others, 2005). Fixed-count adjustments are necessary even if samples are collected using the same method because standardized laboratory subsampling procedures result in a wide range of individuals extracted from each sample, which differentially affects taxa richness across samples (Vinson and Hawkins, 1996).

Although three different protocols were used to collect the macroinvertebrate data compiled for this study, the differences were considered negligible after the adjustments were made as described in the previous paragraph. For example, all methods targeted riffle habitats, collected multiple samples along a stream reach, scrubbed the overlying substrate within a defined area, disturbed the underlying substrate, and used a similar mesh size ( 425 or 500 microns). The primary difference among field methods was the area of stream bottom sampled (FS Hess sampler, $4.6 \mathrm{ft}^{2}$; FS, $8 \mathrm{ft}^{2}$; USGS, $13.5 \mathrm{ft}^{2}$ ). Carlisle and Hawkins (2008) recently aggregated data collected with different sampled areas of stream bottoms (ranged from 7.8 to $\left.13.5 \mathrm{ft}^{2}\right)$ and found that the ratio of observed (O) taxa to those expected $(\mathrm{E})(\mathrm{O} / \mathrm{E})$ values for samples collected from smaller areas were negatively biased by 0.06 units (a relatively small amount). Considering the results of Carlisle and Hawkins (2008), the lower end of any bias in O/E values evaluated in this study likely is around 0.06 units, although 

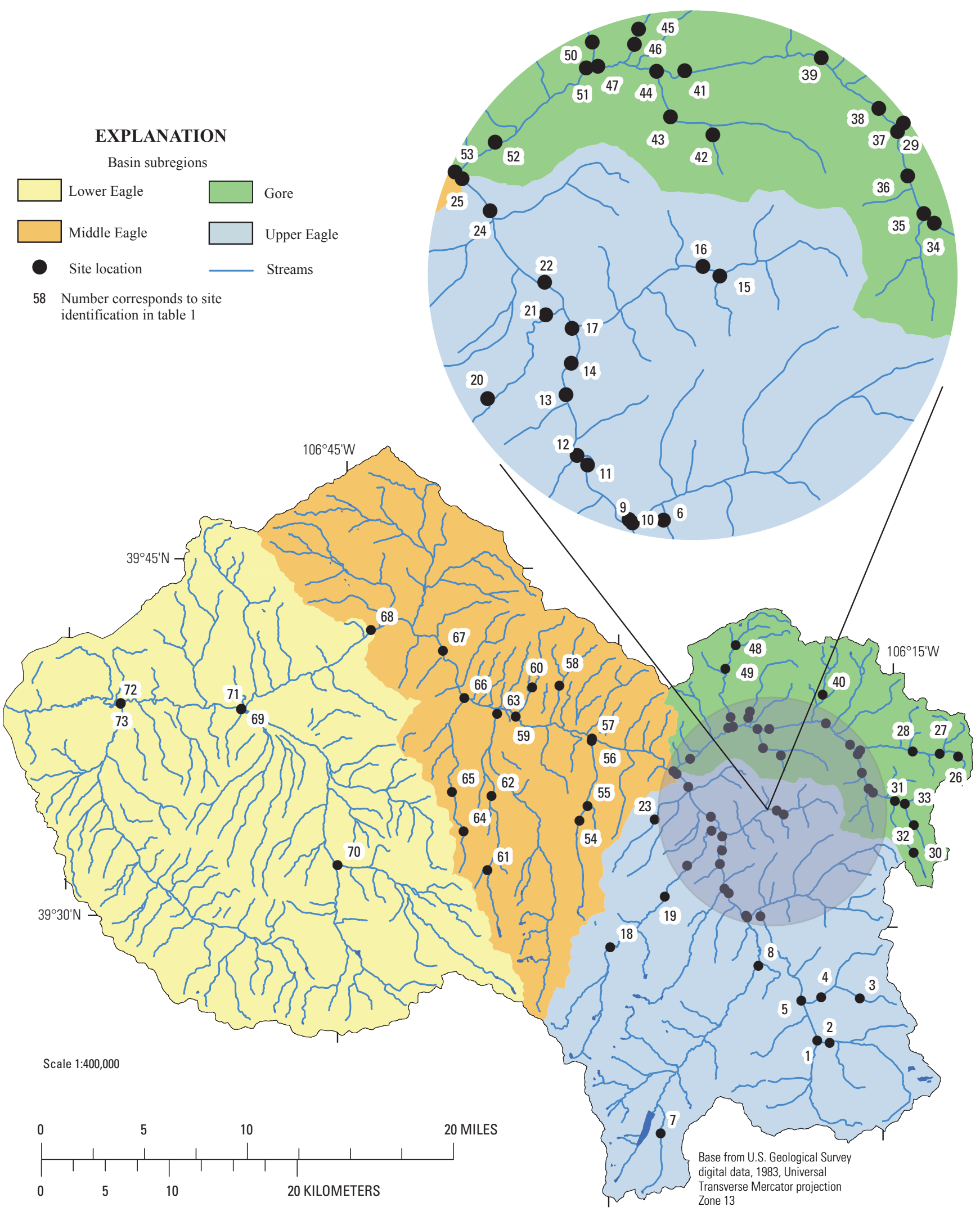

Figure 2. Selected sites and Eagle River watershed subregions, Colorado, 2000-07. 
[ID, identification; latitude and longitude in degrees, minutes, and seconds; NAVD 88, North American Vertical Datum of 1988; ft, feet; mi² , square miles; FS, U.S. Forest Service; USGS, U.S. Geological Survey; O/E, ratio of observed to expected macroinvertebrate taxa; --, not applicable]

\begin{tabular}{|c|c|c|c|c|c|c|c|c|c|c|c|c|c|c|}
\hline $\begin{array}{l}\text { Site ID } \\
\text { (fig. 2) }\end{array}$ & Station name & Station ID & Latitude & Longitude & $\begin{array}{l}\text { Elevatio } \\
\text { n NAVD } \\
88 \text { (ft) }\end{array}$ & $\begin{array}{l}\text { Drainag } \\
\text { e area } \\
\left(\mathrm{mi}^{2}\right)\end{array}$ & Basin group & Site class & Data source & $\begin{array}{l}\text { Number of years } \\
\text { macroinvertebrate samples } \\
\text { collected } 2000-07\end{array}$ & $\begin{array}{l}\text { Most } \\
\text { recent } O / E \\
\text { value }\end{array}$ & $\begin{array}{c}\text { Mean } \\
\text { O/E }\end{array}$ & $\underset{\text { O/E }}{\text { Minimum }}$ & $\begin{array}{c}\text { Maximun } \\
\text { O/E }\end{array}$ \\
\hline 1 & East Fork Eagle River above Colorado Trail Bridge & EFER-R1-1.06 & $\begin{array}{ll}39^{\circ} 25^{\prime} 11^{\prime \prime} \\
30^{\circ} 25^{5} 15^{\prime}\end{array}$ & $\begin{array}{ll}106^{\circ} 18^{\prime} 07^{\prime \prime} \\
106^{\circ}\end{array}$ & 9,326 & & Upper Eagle & Mixed & FS & 2005 & & -- & -- & \\
\hline 2 & $\begin{array}{l}\text { South Fork Eagle River above East Fork } \\
\text { Pearl }\end{array}$ & SFER-R1-0.08 & $39^{\circ} 25^{\prime} 15^{\prime \prime}$ & $106^{\circ} 18^{\prime} 48^{\prime \prime}$ & 9,304 & 19.0 & Upper Eagle & Mixed & FS & 2005 & 0.65 & - & -- & -- \\
\hline & Pearl Creek approximately 1.4 miles from mouth & PERL-R1-2.37 & $39^{\circ} 27^{\prime} 04^{\prime \prime}$ & $106^{\circ} 16^{6} 32^{\prime \prime}$ & 10,238 & 2.44 & Upper Eagle & Undeveloped & FS & 2005 & 1.05 & -- & -- & \\
\hline 年 & $\begin{array}{l}\text { Resolution Creek above Pearl Creek } \\
\text { Eagle Piver Belogw Resolytion Creeek }\end{array}$ & $\begin{array}{l}\text { RESL-R1-1.680 } \\
\text { Refl- }\end{array}$ & $\begin{array}{ll}39^{\circ} 27^{\prime} 06^{\prime \prime \prime} \\
30^{\circ} 26^{\prime} 57^{\prime \prime}\end{array}$ & $\begin{array}{ll}106^{\circ} 8^{\prime} 37^{\prime \prime} \\
106^{\circ} 19^{1} 42^{\prime \prime}\end{array}$ & 9,546 & 11.5 & 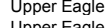 & Adjacent road or highway, or both & FS & 2006 & 0.87 & -- & -- & \\
\hline & Eagle River Below Resolution Creek & EAGL-R1-95.27 & 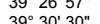 & 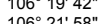 & 9,206 & 58.7 & Upper Eagle & Mixed & FS & 2005 & 1.06 & - & & \\
\hline 栗 & $\begin{array}{l}\text { Eagle River at Red Cliff } \\
\text { East Fork Homestake Creek above County Line }\end{array}$ & 9063000 & $\begin{array}{ll}39^{\circ} 30^{\circ} 30^{\prime \prime} \\
39^{\circ} 214^{\prime \prime} 14\end{array}$ & 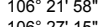 & 8,654 & 70.0 & Upper Eagle & & USGS & 2000,2001 & & 0.99 & 0.92 & 1.06 \\
\hline 8 & $\begin{array}{l}\text { East Fork Homestake Creee above County Line } \\
\text { Homestake Creek near Red Cliff }\end{array}$ & 9064500 & $\begin{array}{ll}3 \\
39^{\circ} 28^{\prime} 24^{\prime \prime}\end{array}$ & 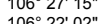 & 10,415 & 5.3 & $\begin{array}{l}\text { Upper Eagle } \\
\text { naner }\end{array}$ & Undeveloped & FS & 2006 & & ( & ( & -- \\
\hline${ }_{9}^{8}$ & Eagle River above Homestake Creek & EAGL-85.162 & $39^{\circ} 30^{\circ} 26^{\prime \prime}$ & $106^{\circ} 22^{\prime} 43^{\prime \prime}$ & $\begin{array}{l}8,103 \\
855\end{array}$ & $\begin{array}{l}58.2 \\
100.2\end{array}$ & $\begin{array}{l}\text { Opper Eagle } \\
\text { Unpoer Eale }\end{array}$ & $\begin{array}{l}\text { Adjacent road or highway, or both } \\
\text { Mixed }\end{array}$ & $\begin{array}{l}\text { USGS } \\
\text { FS }\end{array}$ & 2000 & 年 & - & & - \\
\hline 10 & $\begin{array}{l}\text { Eagle River below Homestake Creek near Red Cliff } \\
\text { E. }\end{array}$ & 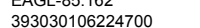 & ${ }_{33^{\circ} 30^{\prime} 30^{\prime \prime}}$ & $106^{\circ} 22^{\prime} 47^{\prime \prime}$ & 8.820 & 164.1 & Uvpere Eacle & Mixed & $\begin{array}{l}\text { FS } \\
\text { USGS }\end{array}$ & 2000,2001 & & 1.19 & 1.19 & 1.19 \\
\hline 11 & Eagle River upstream of Belden & AGL-82.442 & $39^{\circ} 31^{\prime} 27^{\prime \prime}$ & $106^{\circ} 23^{\prime} 45^{\prime \prime}$ & 8.398 & 168.9 & & Mixed & & 2007 & & & & 1.0 \\
\hline 12 & Eagle River upstream of Fall Creek & EAGL-81.615 & $39^{\circ} 31^{\prime} 37^{\prime \prime}$ & $106^{\circ} 23^{\prime} 59^{\prime \prime}$ & 8,344 & 169.4 & $\begin{array}{l}\text { Upper Eagle } \\
\text { Upal }\end{array}$ & Mixed & FS & 2007 & 0.39 & -- & -- & -- \\
\hline 13 & Eagle River below Bishop Gulch & EAGL-79.449 & $39^{\circ} 32^{\prime} 41^{\prime \prime}$ & $106^{\circ} 24^{\prime} 16^{\prime \prime}$ & 8,156 & 184.9 & Upper Eagle & Mixed & FS & 2007 & 0.46 & -- & -- & -- \\
\hline 14 & Eagle River near Minturn & 09064600 & $39^{\circ} 33^{\prime} 14^{\prime \prime}$ & $106^{\circ} 24^{\prime} 07^{\prime \prime}$ & 8,078 & 186.0 & Upper Eagle & Mixed & USGS & 2000 & 0.98 & -- & -- & - \\
\hline 15 & $\begin{array}{l}\text { Unnamed tributary to Two Elk Creek } \\
\text { Twwo Ele Creek helowTea Cuy Bow }\end{array}$ & $\begin{array}{l}\text { EARL-R1-0.191 } \\
\text { TOEL-R1.5197 }\end{array}$ & $\begin{array}{ll}39^{\circ} 34^{4} 49^{\prime \prime} \\
33^{\circ} 34^{\circ} 5 n^{\prime \prime}\end{array}$ & 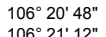 & 9,505 & 1.5 & $\begin{array}{l}\text { Upper Eagle } \\
\text { Utuper Eagle }\end{array}$ & $\begin{array}{l}\text { Mixed } \\
\text { Mixixd }\end{array}$ & 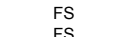 & $\begin{array}{l}2003 \\
2004 \\
2005\end{array}$ & 0.81 & $-\overline{10}$ & $\ddot{0.94}$ & $\therefore$ \\
\hline & $\begin{array}{l}\text { Two Elk Creek below Tea Cup Bowl } \\
\text { Eagle River above Two Elk Creek }\end{array}$ & $\begin{array}{l}\text { TOEL-R1-5.197 } \\
\text { EAGL-76.850 }\end{array}$ & $\begin{array}{ll}39^{\circ} 34^{4} 58^{\prime \prime} \\
39^{\circ} 33^{\prime} 51 "\end{array}$ & $\begin{array}{ll}106^{\circ}{ }^{\circ} 1^{\prime} 12^{\prime \prime} \\
100^{\circ} 24^{\prime} 09^{\prime \prime}\end{array}$ & $\begin{array}{l}9,220 \\
8014\end{array}$ & $\begin{array}{r}7.7 \\
186.7\end{array}$ & $\begin{array}{l}\text { Upper Eagle } \\
\text { Upoper Eagle }\end{array}$ & $\begin{array}{l}\text { Mixixed } \\
\text { Mixed }\end{array}$ & $\begin{array}{l}\text { FS } \\
\text { FS }\end{array}$ & $\begin{array}{l}2004,2005 \\
2007\end{array}$ & 1.08 & 1.01 & 0.94 & 1.08 \\
\hline 18 & $\begin{array}{l}\text { Eagle River above Two Elk Creek } \\
\text { Cross Creek near Reeds Meadow }\end{array}$ & $\begin{array}{l}\text { EALL-76.850 } \\
\text { CROS-R3-15.51 }\end{array}$ & $\begin{array}{lll}39^{\circ} 333^{\prime} 51^{\prime \prime} \\
39^{\circ} 9^{\prime} 05^{\prime \prime}\end{array}$ & $\begin{array}{l}106^{2} 24^{\circ} 09^{\prime \prime} \\
100^{\circ} 30^{\prime} 10^{\prime \prime}\end{array}$ & $\begin{array}{l}8,014 \\
9870\end{array}$ & $\begin{array}{l}186.7 \\
16.13\end{array}$ & $\begin{array}{l}\text { ODper Eagle } \\
\text { Uopoer Eagle }\end{array}$ & $\begin{array}{l}\text { Mixed } \\
\text { Undeveloped }\end{array}$ & $\begin{array}{l}\text { FS } \\
\text { FS }\end{array}$ & $\begin{array}{r}2007 \\
20052006,2007\end{array}$ & $\begin{array}{l}0.52 \\
1.02\end{array}$ & 104 & 102 & 109 \\
\hline 199 & 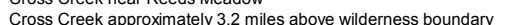 & $\begin{array}{lll} & \end{array}$ & 390 $30^{\circ} 31^{1} 1^{\prime \prime \prime}$ & $106^{\circ} 27^{\prime 1} 14^{\prime \prime}$ & 列 & $\begin{array}{l}28.42 \\
28.42\end{array}$ & 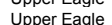 & Undevelooed & Fs & & & 1.04 & & 1.00 \\
\hline 20 & Cross Creek above wilderness boundary & CROSS_01R & $39^{\circ} 32^{\prime} 35^{\prime \prime}$ & $106^{\circ} 26^{\prime} 03^{\prime \prime}$ & 8,682 & 32.57 & Upper Eagle & Undeveloped & & 2005 & & $\ldots$ & -- & $\ldots$ \\
\hline 21 & Cross Creek near Minturn & 09065100 & $39^{\circ} 34^{\prime} 05^{\prime \prime}$ & $106^{\circ} 24^{\prime} 43^{\prime \prime}$ & 7,992 & 34.2 & Upper Eagle & Mixed & USGS & 2000 & 1.05 & -- & -- & -- \\
\hline 22 & Eagle River below Cross Creek & EAGL-74.978 & $39^{\circ} 34^{\prime} 39^{\prime \prime}$ & $106^{\circ} 24^{4} 47^{\prime \prime}$ & 7,917 & 238.4 & Upper Eagle & Mixed & FS & 2007 & 0.69 & -- & -- & -- \\
\hline & West Grouse Creek below pack trail crossing & WGRO-2.794 & $39^{\circ} 34^{4} 30^{\prime \prime}$ & & 9,391 & 4.7 & & Mixed & FS & 2007 & 0.95 & - & -- & -- \\
\hline $\begin{array}{l}24 \\
25\end{array}$ & $\begin{array}{l}\text { Eagge River below Game Creek } \\
\text { Eagle River above Gore Creek near Minturn }\end{array}$ & $\begin{array}{l}\text { EAGL-711.778 } \\
393627106264000\end{array}$ & $\begin{array}{ll}39^{\circ} 35^{\circ} 54^{\prime \prime} \\
39^{\circ} 67^{\prime} 27^{\prime \prime}\end{array}$ & $\begin{array}{ll}106^{\circ} 6^{\circ} \\
106^{\circ} 23^{\prime \prime} \\
0^{\prime \prime}\end{array}$ & $\begin{array}{l}7,804 \\
7,730\end{array}$ & $\begin{array}{l}258.6 \\
259.9\end{array}$ & $\begin{array}{l}\text { Upper Eagle } \\
\text { Uopoer Eagle }\end{array}$ & $\begin{array}{l}\text { Mixixed } \\
\text { Mixed }\end{array}$ & $\begin{array}{l}\text { FS } \\
\text { USGS }\end{array}$ & $\begin{array}{l}2007 \\
2000\end{array}$ & $\begin{array}{l}0.49 \\
1.04\end{array}$ & $-\overline{-}$ & -- & $-\overline{-}$ \\
\hline 26 & $\begin{array}{l}\text { Ealie River above Gore Creeen nears Wilinurn } \\
\text { Gore Creek near Red Buffalo Pass }\end{array}$ & $\begin{array}{l}39302700224000 \\
\text { GORE-R9-27.102 }\end{array}$ & $\begin{array}{ll}39^{\circ} 37^{\prime} 21^{\prime \prime} \\
\end{array}$ & $106^{\circ} 11^{\prime 2} 20^{\prime \prime}$ & 10,636 & $\begin{array}{r}259.9 \\
139\end{array}$ & $\begin{array}{l}\text { Upper Eagle } \\
\text { Gore }\end{array}$ & $\begin{array}{l}\text { Mixed } \\
\text { Undeveloped }\end{array}$ & FS & 2005 & $\begin{array}{l}1.04 \\
1.02\end{array}$ & $-\overline{-}$ & & $-\overline{-}$ \\
\hline 27 & Gore Creek approximately 3.5 miles above wilderness boundary & $\begin{array}{l}\text { GORE-RG-27.102 } \\
\text { GORE-R8-25.38 }\end{array}$ & $\begin{array}{ll}39^{\circ} 37^{\prime} 28^{\prime \prime} \\
39^{\prime}\end{array}$ & $\begin{array}{ll}106^{\prime} 12^{\prime} 20^{\prime \prime} \\
100^{\circ}\end{array}$ & $\begin{array}{l}10,636 \\
10,172\end{array}$ & $\begin{array}{l}1.39 \\
5.46\end{array}$ & $\begin{array}{l}\text { Gore } \\
\text { Gore }\end{array}$ & $\begin{array}{l}\text { Undeveloped } \\
\text { Undeveloped }\end{array}$ & FS & 2004, 2005, 2007 & $\begin{array}{l}1.02 \\
1.09\end{array}$ & $1 . \overline{14}$ & 1.09 & $\overline{1.16}$ \\
\hline 28 & Gore Creek approximately 2 miles above wilderness boundary & GORE-R7-23.05 & $39^{\circ} 37^{\prime} 33^{\prime \prime}$ & $106^{\circ} 13^{\prime} 49^{\prime \prime}$ & 9,779 & 8.20 & Gore & Undeveloped & FS & $2004,2005,2007$ & 0.95 & 0.95 & 0.87 & 1.02 \\
\hline & Gore Creek at Upper Station near Minturn & 09065500 & $39^{\circ} 37^{\prime} 33^{\prime \prime}$ & $106^{\circ} 16^{\prime} 39^{\prime \prime}$ & 8,600 & 14.4 & Gore & Undeveloped & FS, USGS & $2000,2001,2004,2005,2007$ & 1.09 & 0.98 & 0.87 & 1.09 \\
\hline 30 & Black Gore Creek below Black Lake No. 2 & BGOR-R4-10.64 & $39^{\circ} 33^{\prime} 16^{\prime \prime}$ & $106^{\circ} 13^{\prime} 40^{\prime \prime}$ & 10,339 & 2.1 & Gore & Adjacent road or highway, or both & FS & $2004,2005,2006,2007$ & 0.92 & 0.70 & 0.46 & 0.92 \\
\hline 31 & Black Gore Creek above Polk Creek & BGOR-R3-5.73 & $39^{\circ} 35^{\circ} 26^{\prime \prime}$ & $106^{\circ} 14^{\prime} 45^{\prime \prime}$ & 9,482 & 4.6 & Gore & Adjacent road or highway, or both & FS & 2004, 2005, 2006, 2007 & 0.88 & 0.86 & 0.74 & 1.01 \\
\hline 32 & Polk Creek approximately 1.25 miles above wilderness boundary & POLK-R3-2.90 & $39^{\circ} 34^{\prime} 26^{\prime \prime}$ & $\begin{array}{ll}106^{\circ} 13^{\prime} 42^{\prime \prime} \\
100^{\prime}\end{array}$ & 10,277 & 1.48 & Gore & Undeveloped & FS & $2004,2005,2006$ & 1.05 & 0.94 & 0.85 & 1.05 \\
\hline $\begin{array}{l}33 \\
34\end{array}$ & $\begin{array}{l}\text { Polk Creek above wilderness boundary } \\
\text { Black Gore Creek near Minturn }\end{array}$ & $\begin{array}{l}\text { POLKR-R2-0.97 } \\
09066000\end{array}$ & $\begin{array}{l}39^{\circ} 35^{\prime} 19^{\prime \prime} \\
39^{\circ} 5^{\prime} 47^{\prime \prime}\end{array}$ & $\begin{array}{l}106^{1}{ }^{\circ} 4^{\prime} 12^{\prime \prime \prime} \\
100^{\prime \prime} 15^{\prime} 52^{\prime}\end{array}$ & $\begin{array}{l}9,911 \\
9,150\end{array}$ & $\begin{array}{l}2.46 \\
12.6\end{array}$ & $\begin{array}{l}\text { Gore } \\
\text { Gore }\end{array}$ & $\begin{array}{l}\text { Undeveloped } \\
\text { Adiacent road or highway or both }\end{array}$ & $\begin{array}{l}\text { FS } \\
\text { FS, USGS }\end{array}$ & $\begin{array}{l}2004,2005,2006,2007 \\
2000,2004,2005,2006,2007\end{array}$ & $\begin{array}{l}0.95 \\
0.87\end{array}$ & $\begin{array}{l}1.00 \\
0.79\end{array}$ & $\begin{array}{l}0.95 \\
0.43\end{array}$ & 1.08 \\
\hline $\begin{array}{l}34 \\
35\end{array}$ & $\begin{array}{l}\text { black Gore Creek near Minturn } \\
\text { Timber Creek at mouth }\end{array}$ & $\begin{array}{l}09066000 \\
\text { TIMBER-R1- }\end{array}$ & $\begin{array}{l}39^{\circ} 35^{\circ} 4 r^{\prime \prime} \\
39^{\circ} 58^{\prime} 5{ }^{\prime}\end{array}$ & $\begin{array}{l}106^{1}-55^{\prime \prime \prime} \\
106^{\circ} 16^{\prime \prime} 11^{\prime}\end{array}$ & $\begin{array}{l}9,150 \\
9,119\end{array}$ & $\begin{array}{r}12.6 \\
3.4\end{array}$ & $\begin{array}{l}\text { Gore } \\
\text { Gore }\end{array}$ & $\begin{array}{l}\text { Adjacent roado or highway, or both } \\
\text { Adjacent road or highway, or both }\end{array}$ & $\begin{array}{ll}\text { FS, USGS } \\
\text { FS }\end{array}$ & $\begin{array}{l}2000,2004,2005,2006,200 r \\
2005\end{array}$ & 0.98 & & 0.43 & $\begin{array}{l}1.16 \\
-.\end{array}$ \\
\hline $\begin{array}{l}35 \\
36\end{array}$ & Black Gore Creek at $1-70$ mile post 183 & $\begin{array}{l}\text { BGOR-R1-1.83 } \\
\text { B } 0.4444\end{array}$ & - & $106^{\circ} 16^{\prime} 34^{\prime \prime}$ & 8,921 & $\begin{array}{l}3.4 \\
18.6\end{array}$ & Gore & 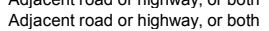 & FS & 2004, 2005, 2006, 2007 & 1.01 & 0.96 & 0.87 & 1.01 \\
\hline 37 & Black Gore Creek near Vail & 09066050 & $39^{\circ} 37^{\prime} 24^{\prime \prime}$ & $106^{\circ} 16^{\prime} 47^{\prime \prime}$ & 8,570 & 19.6 & Gore & Adjacent road or highway, or both & USGS & 2000,2001 & 0.94 & 0.94 & 0.94 & 0.94 \\
\hline 38 & Gore Creek at Bighorn Park & GORE-R4-17.37 & $39^{\circ} 37^{\prime} 48^{\prime \prime}$ & $106^{\circ} 17^{\prime} 15^{\prime \prime}$ & 8,516 & 35.4 & Gore & Urban & FS & $2005,2006,2007$ & 0.56 & 0.61 & 0.56 & 0.70 \\
\hline 39 & Gore Creek above Katsos & 393836106182500 & $39^{\circ} 38^{\prime} 36^{\prime \prime}$ & $106^{\circ} 18^{\prime 2} 25^{\prime \prime}$ & 8,355 & 47.6 & Gore & Urban & $\mathrm{FS}$, USGS & $2000,2004,2005,2006,2007$ & 0.56 & 0.76 & 0.56 & 1.05 \\
\hline 40 & Booth Creek approximately 1.0 mile above wilderness boundary & booth_R3_02R-2.5407 & $\begin{array}{ll}39^{\circ} 39^{\prime} 53^{\prime \prime} \\
30^{\circ} 30^{\circ} 25^{\prime}\end{array}$ & $106^{\circ} 18^{4} 47 " 1 "$ & 9,317 & 4.10 & Gore & Undeveloped & 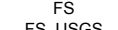 & 2005 & 1.02 & 072 & 063 & $\because 0$ \\
\hline 41 & $\begin{array}{l}\text { Gore Creek downstream of Pulis Bridge } \\
\text { Unnamed tributary to Mill Creek draining Northeast Bowl }\end{array}$ & $\begin{array}{l}393825106213400 \\
\text { NFBL-R1-0.374 }\end{array}$ & $\begin{array}{ll}39^{\circ} 38^{8} 25^{\prime \prime} \\
39^{\circ} 37^{\prime \prime} 17^{\prime \prime}\end{array}$ & $\begin{array}{l}106^{\circ}{ }^{\circ} 1^{3} 34^{\prime \prime} \\
100^{\circ} 21^{\prime} 01^{\prime \prime}\end{array}$ & 8,236 & $\begin{array}{c}58.7 \\
1.1\end{array}$ & $\begin{array}{l}\text { Gore } \\
\text { Gore }\end{array}$ & $\begin{array}{l}\text { Urban } \\
\text { Mixed }\end{array}$ & $\begin{array}{l}\text { FS, USGS } \\
\text { FS }\end{array}$ & $2000,2004,2005,2006,2007$ & 0.63 & 0.72 & 0.63 & \\
\hline & Unnamed tributary to Mill Creek near Mid Vail & $\begin{array}{l}\text { NEBLR-R1-0.374 } \\
\text { PTAR-R1-0.066 }\end{array}$ & $39^{\circ} 37^{\prime} 36^{\prime \prime}$ & $106^{\circ} 21^{\prime} 59^{\prime \prime}$ & 8,878 & 1.1 & $\begin{array}{l}\text { Gore } \\
\text { Gore }\end{array}$ & $\begin{array}{l}\text { Mixed } \\
\text { Mixed }\end{array}$ & FS & $\begin{array}{l}2003,2005 \\
2005\end{array}$ & 0.54 & 0.09 & 0.05 & 0.72 \\
\hline 44 & Mill Creek near Vail & 700 & $38^{\prime 2} 24^{\prime \prime}$ & & 8,210 & 7.5 & Gore & $\begin{array}{l}\text { Milea } \\
\text { Mixed }\end{array}$ & USGS & 2000,2001 & 0.36 & 0.65 & 0.36 & 0.94 \\
\hline & Middle Creek approximately 0.5 miles a & MIDL-F & & $106^{\circ} 22^{\prime} 44^{\prime \prime}$ & 8,644 & 5.82 & Gore & Undeveloped & FS & 2005 & 1.02 & $\therefore$ & $\cdots$ & 0.54 \\
\hline 46 & Middle Creek above wilderness bound & MIDL-F & $39^{\circ} 38^{\prime} 52^{\prime \prime}$ & $106^{\circ} 22^{\prime} 50^{\prime \prime}$ & 8,419 & 5.90 & Gore & Undeveloped & FS & 200 & 1.09 & -- & -- & -. \\
\hline 47 & Gore Creek at Low & 09066 & $39^{\circ} 38^{\prime} 28^{\prime \prime}$ & $106^{\circ} 23^{\prime} 37^{\prime \prime}$ & 8,060 & 77.1 & Gore & Urban & USGS & 20 & & -- & -- & -- \\
\hline 48 & & & & & & 2.4 & & $y$, or both & FS & & & -- & -- & -- \\
\hline 49 & Sandstone Creek Road & RDSS- & $\begin{array}{l}39^{\circ} 40^{\circ} 55^{\prime \prime} \\
39^{\circ} 33 \prime^{\prime} 53\end{array}$ & $\begin{array}{ll}106^{\circ} 24^{0} 07^{\prime \prime} \\
106^{\prime} 23^{\prime} 47^{\prime \prime}\end{array}$ & $\begin{array}{l}9,206 \\
8238\end{array}$ & 7.4 & Gore & $\begin{array}{l}\text { Adjacent road or highway, or both } \\
\text { Adiacentroag }\end{array}$ & FS & 20 & 1.02 & - & - & $-\overline{-}$ l \\
\hline & $\begin{array}{l}\text { stone Creee at Town of Vali city limit } \\
\text { k below wastewater treatment plant }\end{array}$ & & & & 8,050 & $\begin{array}{l}13.8 \\
91.1\end{array}$ & $\begin{array}{l}\text { Gore } \\
\text { Gore }\end{array}$ & $\begin{array}{l}\text { Adjacent road or highway, or both } \\
\text { Urban }\end{array}$ & $\begin{array}{l}\text { FS } \\
\text { USGS }\end{array}$ & 20 & & -- & $-\overline{-}$ & -- \\
\hline 52 & Gore Creek at Stephens Park & 3600 & & $106^{\circ} 25^{\prime} 36^{\prime \prime}$ & 7,825 & 100.1 & $\begin{array}{l}\text { Gore } \\
\text { Gore }\end{array}$ & Urban & FS, USGS & $0,2004,200$ & 0. & 0.60 & 0.51 & 0.73 \\
\hline 53 & Gore Creek at mouth near Minturn & & $39^{\circ} 36^{\prime} 34^{\prime \prime}$ & $106^{\circ} 26^{\prime} 50^{\prime \prime}$ & 7,730 & 102.0 & Gore & Urban & USGS & 2000,2001 & 0.66 & 0.74 & 0.66 & 0.81 \\
\hline & derness boundary & B) & & & 9,322 & 7.1 & Middle Eagle & Undeveloped & FS & 2005 & 1.05 & & -- & \\
\hline 55 & Beaver Creek abo & 393501106313200 & $39^{\circ} 35^{\prime} 01 "$ & $106^{\circ} 31^{\prime} 32^{\prime \prime}$ & 8,840 & 8.1 & Middle Ez & Mix & usc & 2000, & 1.09 & 0.90 & 0.72 & 1.09 \\
\hline & Beaver & & & & 7,453 & 14.8 & & & & & & & & \\
\hline $\begin{array}{l}57 \\
58\end{array}$ & Eagle River at Avon & $\begin{array}{l}\text { 09067005 } \\
\text { JUNE-B1-3430 }\end{array}$ & & & 7,410 & 395.0 & & in & 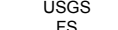 & & 0.90 & 0.84 & 0.78 & 0.90 \\
\hline $\begin{array}{l}58 \\
59\end{array}$ & $\begin{array}{l}\text { June Creee above June Creek Dich } \\
\text { Eagle River at Edwards }\end{array}$ & 393845106353000 & $\begin{array}{ll}39^{\circ} 38^{\prime} 45^{\prime \prime} \\
3\end{array}$ & & $\begin{array}{l}7,1960 \\
7,180\end{array}$ & $\begin{array}{r}5.2 \\
423.8\end{array}$ & $\begin{array}{l}\text { Midali } \\
\text { Middl: }\end{array}$ & $\begin{array}{l}\text { acent road or highway, or both } \\
\text { an }\end{array}$ & USGS & 2000,2001 & & 0.66 & 0.66 & $0 . \overline{6}$ \\
\hline 60 & Berry Cre & & & $106^{\circ} 34^{4} 40^{\prime \prime}$ & & & & & & & & 0.00 & 0.00 & 0.00 \\
\hline 61 & West Lake & & & & & & & & & & & -- & -- & -- \\
\hline & West Lake Creek near Edwards & 06364700 & & & 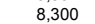 & 11.0 & Mid & or both & 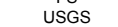 & & & & & -- \\
\hline 63 & Lake Creek nea & & & $106^{\circ} 36^{\prime} 31^{\prime \prime}$ & 7,160 & 49.0 & & & USGS & 2000,2001 & 0.63 & 0.67 & 0.63 & 0.70 \\
\hline 64 & Squaw Creek below w & & & & 9,357 & 2.1 & & Un & FS & & & & & \\
\hline 65 & $\begin{array}{l}\text { Squaw } \mathrm{Cr} \\
\text { Sapwor }\end{array}$ & 年 & & & 8,512 & 4.12 & & & & 2005,2006 & 1.06 & 0.92 & 0.78 & 1.06 \\
\hline 66 & $\begin{array}{l}\text { Squaw Creek at m } \\
\text { Eagle River at Eag }\end{array}$ & & & & 7,122 & & & & $\begin{array}{l}\text { USGS } \\
\text { USGS }\end{array}$ & 20 & & $\overline{-}$ & $\overline{-}$ & $-\overline{-}$ \\
\hline 68 & Eagle River below Milk Creek near I & 00 & $39^{\circ} 42^{\prime}$ & $106^{\circ} 43^{\prime} 33^{\prime \prime}$ & $\begin{array}{l}6,82 \\
6,82\end{array}$ & & Middle Eagle & Adjacent road & 年 & 2000,2001 & & 0.76 & 0.70 & 0.82 \\
\hline & & & & & 6,500 & & & & & & & 0.10 & 0.10 & \\
\hline 70 & East Brush Creek above confluence & 06450700 & $39^{\circ} 32^{\prime 2} 2$ & $106^{\circ} 45^{\prime} 07^{\prime \prime}$ & 7,630 & 32.6 & Lower Eagle & Adjacent road or highway, or & USGS & 2000 & 0.84 & -- & $\ldots$ & \\
\hline 71 & Brush Creek at mouth near Eagle & 393851106503400 & $39^{\circ} 38^{\prime} 5^{\prime \prime \prime}$ & $106^{\circ} 50^{\prime} 34^{\prime \prime}$ & 6,500 & 147.3 & Lower Eagle & Urban & USGS & 2000, 2001 & 0.59 & 0.59 & 0.59 & 0.59 \\
\hline $\begin{array}{l}72 \\
73\end{array}$ & $\begin{array}{l}\text { Eagle River at Gypsum } \\
\text { Gypsum Creek at mouth }\end{array}$ & $\begin{array}{l}090690000 \\
393858106570900\end{array}$ & $\begin{array}{ll}39^{\circ} 39^{\circ} 09^{\circ} 00^{\prime \prime} \\
39^{\circ} 38^{\prime} 58^{\prime \prime}\end{array}$ & $\begin{array}{ll}106^{\circ}{ }^{\circ} 7^{\prime} 16^{\prime \prime \prime} \\
106^{\prime} 57^{\prime} 09^{\prime \prime}\end{array}$ & $\begin{array}{l}6,276 \\
6,280\end{array}$ & $\begin{array}{l}842.0 \\
102.8\end{array}$ & $\begin{array}{l}\text { Lower Eagle } \\
\text { Lower Eagle }\end{array}$ & $\begin{array}{l}\text { Mixed } \\
\text { Urban }\end{array}$ & $\begin{array}{l}\text { USGS } \\
\text { USGS }\end{array}$ & $\begin{array}{l}2000,2001 \\
2000,2001\end{array}$ & $\begin{array}{l}0.47 \\
0.60\end{array}$ & $\begin{array}{l}0.53 \\
0.65\end{array}$ & $\begin{array}{l}0.47 \\
0.60\end{array}$ & $\begin{array}{l}0.58 \\
0.69\end{array}$ \\
\hline
\end{tabular}


the upper limit of indicator-value bias is unknown. Ideally, data comparability using different methods and laboratories would be evaluated from side-by-side comparisons of samples collected from the same sites at the same time (Taylor and others, 2001). Such data for side-by-side comparisons were not available for this study so based on previous studies, it was assumed that the above adjustments improved the comparability of these data. Preferably, results from studies such as this one would be generated from data collected using identical field and laboratory methods, which need to be considered for future monitoring efforts in the ERW.

\section{Assigning Site Classes}

All 73 sites were classified into one of four classes (table 1; undeveloped, adjacent road or highway or both, mixed, and urban) using a combination of riparian land-cover characteristics, examination of topographic maps and aerial imagery, screening for exceedances in water-quality standards, and best professional judgment. Riparian land-cover characteristics were calculated using a geographic information system (GIS) that included watershed-level topographic and landcover data. Stream networks were obtained from the National Hydrography Dataset (U.S. Geological Survey, 2007). Topographic information and digitized vector polygon watershed boundaries were derived from 30-m resolution USGS National Elevation Data (U.S. Geological Survey, 2007). Land-cover data were derived from an enhanced version of the National Land Cover Dataset (Multi-Resolution Land Characteristics Consortium, 2001), a 16-class, 30-m resolution raster dataset derived from multiseason Landsat 5 and Landsat 7 imagery centered on a nominal collection year of 2001. Riparian corridors were delineated with a $100-\mathrm{m}$ buffer around all stream segments upstream from each sampling site and land-cover attributes were calculated. Urban sites initially were classified based on the presence of greater than 1.0 percent urban land cover and no agriculture land cover within the riparian corridor; whereas, sites with both urban and agriculture land cover initially were classified as mixed. Sites with no urban or agriculture land cover initially were classified as undeveloped. Undeveloped sites also were screened for known exceedances of State and Federal water-quality standards and adjusted accordingly (that is, sites that exceeded water-quality standards were removed from the undeveloped site class). Initial classifications were verified and adjusted by examination of USGS 7.5-minute topographic maps and aerial imagery for other types of disturbance within two stream segments upstream from each site. Stream segments were defined by the confluence of upstream tributaries. Other types of disturbance included adjacent roads or highways or both (including native-surfaced roads), unimproved road-stream crossings, hydrologic alteration from reservoirs or diversions, logging, mining, or ski-area development activities. After initial urban and undeveloped site classifications were adjusted, all sites with more than one obvious nearby (that is, within two stream segments) upstream disturbance (for example, mining, channelization, wastewater effluent) were classified as mixed. Final adjustments were made using best professional judgment that was based on historical knowledge of each site and FS field notes. The above classification scheme resulted in 18 undeveloped sites, 15 adjacent road or highway sites or both, 26 mixed sites, and 14 urban sites (table 1). See figure 3 for representative photographs of each site class.

\section{Data Analysis}

An independently developed predictive model (Paul and others, 2005) was applied to calculate a measure of taxonomic completeness for 124 macroinvertebrate samples collected from 73 sites in the ERW. The model calculated taxonomic completeness as the ratio of observed (O) taxa collected at each site to the taxa that were expected (E) to occur at each site in the absence of environmental degradation (Hawkins, 2006). Biological condition (that is, biologically "degraded" or "good") was assessed by setting an O/E threshold value to determine biological degradation. Initial assessments were made and summarized across the four subwatersheds of the ERW: Gore Creek, Upper Eagle, Middle Eagle, and Lower Eagle. Biological condition also was summarized across the four land-cover classes (undeveloped, adjacent road or highway or both, mixed, and urban) and analysis of variance was used to determine whether mean macroinvertebrate $\mathrm{O} / \mathrm{E}$ values were associated with different land-cover classes. Additionally, it statistically was determined which macroinvertebrate taxa occurred more or less frequently at urban sites than predicted using the z-statistic.

\section{Macroinvertebrate Predictive Model}

An independently developed predictive model constructed by the Western Center for Monitoring and Assessment of Freshwater Ecosystems (Utah State University, Logan) was applied to the macroinvertebrate dataset. The predictive model is detailed by Charles P. Hawkins (Western Center for Monitoring and Assessment of Freshwater Ecosystems, Utah State University, Logan) in Paul and others (2005). The model can be accessed by requesting a user name and password from the Western Center for Monitoring and Assessment of Freshwater Ecosystems at http://129.123.10.240/WMCPortal/ DesktopDefault.aspx? tabindex $=0 \&$ tabid $=1$.

The model measures taxonomic completeness represented as the ratio of observed (O) taxa to the taxa that were expected (E) to occur at a site $(\mathrm{O} / \mathrm{E})$ in the absence of environmental degradation (Hawkins 2006). Values of O/E near 1.0 imply that the observed taxa $(\mathrm{O})$ found at a site closely resemble the taxa that were predicted (E) to occur; whereas, $\mathrm{O} / \mathrm{E}$ values $<1.0$ implies some degree of biological degradation. The description of constructing and evaluating predictive models are well documented and detailed elsewhere (Moss and others, 1987; Hawkins and Carlisle, 2001; Clarke and others, 2003; 

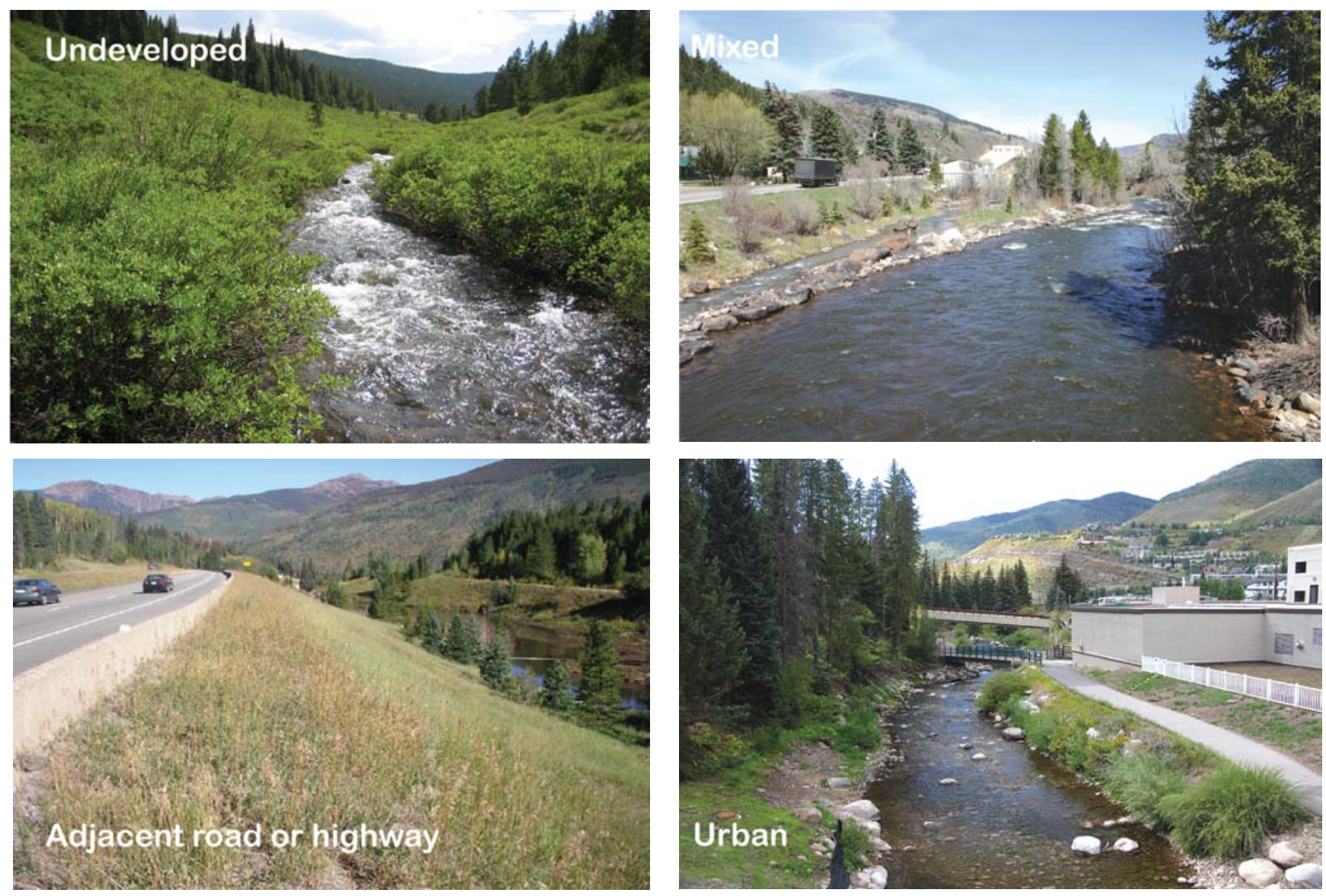

Figure 3. Representative photographs of the four site classes used to evaluate the effect of land cover on biological condition in the Eagle River watershed, Colorado, 2000-07.

Ostermiller and Hawkins, 2004; Van Sickle and others, 2005), and are not detailed herein. However, a brief summary of the model's performance is provided as detailed in Paul and others (2005), as well as how the model was applied to the ERW macroinvertebrate data evaluated in this report.

\section{Predictive Model Description and Performance}

The predictive model (Paul and others, 2005) was constructed from reference site data collected in Colorado (97 sites) using a variety of sampling methods associated with different programs (for example, U.S. Environmental Protection Agency Environmental Monitoring and Assessment Program, Utah State University Western Center for Monitoring and Assessment of Freshwater Ecosystems Science to Achieve Results program, USGS NAWQA, and CDPHE). The model uses three predictor variables (longitude in decimal degrees, mean annual air temperature in ${ }^{\circ} \mathrm{C} \times 10$, and $\log _{10}$ watershed area in $\mathrm{km}^{2}$ ) to generate estimates of $\mathrm{E}$ in which $\mathrm{O}$ was compared. In general, the model was both accurate and precise, and there was little evidence that the model was biased (under or over predicted $\mathrm{E}$ ) towards any major river watershed or ecoregion in Colorado (Paul and others, 2005). Overall, model performance was comparable or better than most macroinvertebrate predictive models in use in the United States or other countries (Paul and others, 2005).

\section{Applying the Predictive Model to the Eagle River Watershed Dataset}

Two steps were necessary before the model was applied to the ERW data. First, the macroinvertebrate identifications in the ERW dataset were harmonized with the operational taxonomic units (OTU) used to develop the model. Second, climatological data were added to the GIS dataset described earlier that were obtained from PRISM (Parameter-elevation Regressions on Independent Slopes Model) datasets, which represent 30-year (1961-90) climate averages (Spatial Climate Analysis Service, 2004). These data were used to calculate the climate-related predictor variable used as part of the model (that is, mean annual air temperature in ${ }^{\circ} \mathrm{C} \times 10$ ). 
The appropriateness of applying the model to the ERW data was evaluated in two ways. First, mean O/E and standard deviation (SD) values calculated from ERW reference sites (undeveloped sites, table 1) were compared to the range of values from the reference sites used to develop the predictive model. Because theoretical $\mathrm{O} / \mathrm{E}$ values from reference sites should equal 1.0, it was determined if ERW reference site $\mathrm{O} / \mathrm{E}$ values statistically were different from 1.0 using a onesample t-test (SPSS, 2005) with a p value of less than 0.05. This statistical test determined whether there was significant bias in predictions of E at ERW sites. A second statistical test (chi-squared test as described in Clarke and others, 2003) was used to determine whether the combined predictor variable distributions for each ERW site were within the experience of the model, which provided assurance that the predictive model was appropriate for assessing the biological condition of ERW sites.

The model produced unbiased estimates of $\mathrm{E}$ when applied to the ERW data, as mean (1.02) and SD (0.07) of O/E values from ERW reference sites were well within the range of mean (1.00) and SD (0.17) values of the reference sites used to develop the predictive model. Additionally, mean O/E values of ERW reference sites were not statistically distinguishable from $1.0(\mathrm{p}=0.24)$ and mean $\mathrm{O} / \mathrm{E}$ reference site values from the model also were equal to 1.0 (Paul and others, 2005). Furthermore, of the 73 sites included in this study (table 1), none of the combined predictor variable distributions (longitude in decimal degrees, mean annual air temperature in ${ }^{\circ} \mathrm{C} \times 10$, and $\log _{10}$ watershed area in $\mathrm{km}^{2}$ ) were outside the range of the model; therefore, all sites were considered for further analysis.

\section{Assessing Biological Condition}

The intent of this investigation was to assess the biological condition of selected ERW sites using macroinvertebrate $\mathrm{O} / \mathrm{E}$ values; therefore, a threshold was set to define two levels of biological condition: sites where $\mathrm{O} / \mathrm{E}$ was greater than $>0.80$ were considered in "good" biological condition, and sites where $\mathrm{O} / \mathrm{E}$ was less than $<0.80$ were considered biologically "degraded." Several methods for setting thresholds to define degraded biological condition have been suggested (Barbour and others, 1999; Hemsley-Flint, 2000; Clarke and others, 2003; Van Sickle and others, 2005). Herein, it was assumed that a 20-percent loss of expected taxa $(\mathrm{O} / \mathrm{E}<0.80)$ was ecologically meaningful, corresponding with the early stages of environmental degradation described by Davies and Jackson (2006). From a statistical standpoint, this threshold $(0.80)$ also was near the 10th percentile of model reference site $\mathrm{O} / \mathrm{E}$ values (0.79); therefore, sites with $\mathrm{O} / \mathrm{E}$ values below the 10th-percentile value would be statistically distinguishable from reference sites $(p>0.01)$. Using the 10th percentile value as a threshold for assessing biological condition in this study, reasonably balances between type I and type II statistical errors (that is, balances between calling a reference site degraded when it is not, and calling a degraded site reference when it is not) while indicating an ecologically meaningful loss of taxa $(\mathrm{O} / \mathrm{E}<0.80)$. It is emphasized that site assessments of biological condition ("good" or "degraded") made herein are unique to this study and are not directly comparable to assessments made based on other approaches, where degraded biological conditions are defined using different indicators, thresholds, or methods.

Biological assessments were summarized by grouping sites into different subregions of the ERW (fig. 2, table 1) as defined hereafter as Gore Creek, Upper Eagle, Middle Eagle, and Lower Eagle. The Gore Creek subregion was inclusive of the entire Gore Creek watershed. The Upper Eagle included the Eagle River and all tributaries upstream from the Gore Creek confluence. The Middle Eagle was defined by the Eagle River and all tributaries between the Gore Creek confluence downstream to Wolcott. Finally, the Lower Eagle included the Eagle River and all tributaries from Wolcott downstream to the confluence with the Colorado River.

\section{Comparing Biological Condition Among Site Classes}

Test statistics (F, p) were calculated from analysis of variance (ANOVA) to determine if site class (undeveloped, adjacent road or highway or both, mixed, and urban) accounted for variability in mean $\mathrm{O} / \mathrm{E}$ values using the most recent sample collected at each site (table. 1). Additionally, post-hoc tests of pairwise differences were conducted between site classes based on Bonferroni adjustments of experimental error (SPSS ver. 13.0, SPSS, 2005). ANOVA and post-hoc tests were considered significant if $\mathrm{p}<0.05$.

\section{Annual Variability in Observed/Expected}

The FS initiated a macroinvertebrate monitoring program in 2004 at selected sites in the Gore Creek watershed in response to stakeholder concerns related to potential effects of excessive sediment deposition along Black Gore and Gore Creeks (Healy, 2008). As a result, 3 to 4 macroinvertebrate samples were collected at 13 sites annually within the Gore Creek watershed between 2004 and 2007. Four of these sites were previously sampled by the USGS in 2000. Additionally, an undeveloped site on Cross Creek was sampled during 2004, 2005, and 2007 by FS. The combination of these annual samples provided the opportunity to examine annual variability in O/E values over a 3-5 year period among 14 sites (undeveloped sites 18, 27, 28, 29, 32, 33, adjacent road and /or highway (Black Gore) sites 30, 31, 34, 36, urban (Gore) sites $38,39,41,52)$. The coefficient of variation $(\mathrm{CV}=[100 \times$ standard deviation/mean]) of $\mathrm{O} / \mathrm{E}$ values was used as a measure of the magnitude of annual variability. 


\section{Observed and Expected Macroinvertebrate Taxa Occurrences at Urban Sites}

Macroinvertebrate taxa that were observed more or less frequently than predicted by the model at urban sites were determined following the methods of Carlisle and others (2008) and Carlisle and Hawkins (2008), by recording the occurrence frequencies $\left(\mathrm{O}_{\mathrm{f}}\right)$ of all observed taxa among urban sites, and by calculating expected frequencies $\left(\mathrm{E}_{\mathrm{f}}\right)$ of occurrence by summing the predicted probabilities of capture for each taxon among all urban sites. If the null hypothesis was true for a given taxon $\left(\mathrm{O}_{\mathrm{f}}=\mathrm{E}_{\mathrm{f}}\right)$, then it was observed as frequently as it was predicted; however, $\mathrm{O}_{\mathrm{f}}>$ or $\left\langle\mathrm{E}_{\mathrm{f}}\right.$ indicates which taxa are observed more or less frequently than predicted among sites. Analysis was limited to the most recent sample collected at each of the 14 urban sites (table 1). The null hypothesis was tested for each taxon using the $\mathrm{z}$-statistic, where $\left.\mathrm{z}=\left(\mathrm{O}_{\mathrm{f}}-\mathrm{E}_{\mathrm{f}}\right) / \sqrt{\operatorname{Variance}\left(\mathrm{O}_{\mathrm{f}}\right.}\right)$ (Carlisle and Hawkins, 2008). Taxa with $|z|>1.96$ were considered to be tolerant increasers if $\mathrm{O}_{\mathrm{f}}>\mathrm{E}_{\mathrm{f}}$ or intolerant decreasers if $\mathrm{O}_{\mathrm{f}}<\mathrm{E}_{\mathrm{f}}$. Additionally, taxa that were observed as often as predicted ( \pm one site) were considered to be tolerant of the environmental conditions found at urban classified sites. Reported results were restricted to only those taxa that were predicted or observed at one-half or more of the 14 urban sites. See Carlisle and Hawkins (2008) for details to calculate Variance $\left(\mathrm{O}_{\mathrm{f}}\right)$.

\section{Macroinvertebrate-Based Assessment of Biological Condition}

The number of sites available for assessment varied by subregion as did sampling intensity. The Gore Creek subregion was the most heavily sampled (28 sites, 65 samples), followed by the Upper Eagle ( 25 sites, 30 samples), Middle Eagle (15 sites, 21 samples), and the Lower Eagle (5 sites, 8 samples) (fig. 4). Overall, 55 percent of sites (40 sites) were considered in good biological condition; whereas, 30 percent (22 sites) were biologically degraded. The additional 15 percent of sites (11 sites) had $\mathrm{O} / \mathrm{E}$ values that ranged both above and below the threshold $(\mathrm{O} / \mathrm{E}<0.80)$ depending on the year sampled, and mean values for six of those sites were below the threshold. Considering these 6 sites, a total of 38 percent ( 28 sites) were considered biologically degraded. Also note that of the 73 sites, 36 percent ( 25 sites) have not been sampled since 2000 or 2001, so it is likely that assessments may not reflect current biological condition at some of these sites. Nonetheless, site assessments are considered a screening tool for determining where in the ERW biological conditions are good or degraded until further investigation confirms otherwise.

These results may underestimate actual taxa loss and sensitivity of $\mathrm{O} / \mathrm{E}$ values to biological degradation. Sites were considered biologically degraded when more than 20 percent of taxa expected to occur at a site were not observed $(\mathrm{O} / \mathrm{E}<0.80)$. By necessity, the model was constructed with relatively coarse taxonomic resolution (for example, taxonomy used in this analysis mostly consisted of genera, some families, midges at subfamily, and few species) (Paul and others, 2005). As a result, most taxonomic units represented more than one species, which likely varied in their individual tolerance to stressors. Such cases can result in O/E models that are less sensitive to some environmental stressors than would be observed if species level data are used (Hawkins and others, 2000).

\section{Site Class and Biological Condition}

Site classes were determined primarily from adjacent land-cover characteristics. Analysis of variance determined that mean $\mathrm{O} / \mathrm{E}$ values varied significantly among site classes $(\mathrm{F}=12.415, \mathrm{p}<0.001)$ based on the most recent samples collected at each site. Urban (mean $\mathrm{O} / \mathrm{E}=0.69)$ and mixed site classes (mean $\mathrm{O} / \mathrm{E}=0.76$ ) exhibited the most degraded biological condition (that is, the lowest mean $\mathrm{O} / \mathrm{E}$ values); whereas, sites in the adjacent road or highway or both class (hereafter, ARH; mean $\mathrm{O} / \mathrm{E}=0.92$ ) were near conditions found at undeveloped sites (mean $\mathrm{O} / \mathrm{E}=1.02$ ) (fig. 5). In general, most degraded sites were located along the main stem Eagle River and lower Gore Creek; whereas, sites located at the higher altitudes in the watershed were in relatively good biological condition regardless of site class (fig. 6). Additionally, results remained similar when urban classified sites along lower Gore Creek were removed from the analysis.

In this study, macroinvertebrate communities at urban sites were most severely degraded (that is, these sites had the lowest mean $\mathrm{O} / \mathrm{E}$ values compared to other site classes), a result that is consistent with many other studies (Paul and Meyer, 2001, and citations within). Carlisle and Meador (2007) and Carlisle and others (2008) reported similar results using $\mathrm{O} / \mathrm{E}$ and a similar degradation threshold; however, they classified urban sites differently. In those two studies, sites were classified as urban when upstream watersheds had greater than 10- and 25-percent urban-land cover, respectively. In the study herein, the highest estimates of watershed urbanland cover did not exceed 2.5 percent, yet similar patterns of biological impairment were observed. Most urban-land cover in the ERW is concentrated in or near riparian areas and can account for as much as 35 percent in some segments (Bledsoe and others, 2005). A recent assessment of wadeable streams in the United States (U.S. Environmental Protection Agency, 2006) indicated riparian vegetated cover disturbance as one of the main stressors increasing the risk of macroinvertebrate impairment and taxa loss in the Western United States. Other primary stressors that increased the risk of biological impairment in western streams included nitrogen, phosphorus, and suspended sediment concentrations. All of these stressors identified in the wadeable streams report (U.S. Environmental Protection Agency, 2006) were independently identified by others (for example, Bledsoe and others, 2005) as concerns affecting the biological integrity of streams in the ERW. 


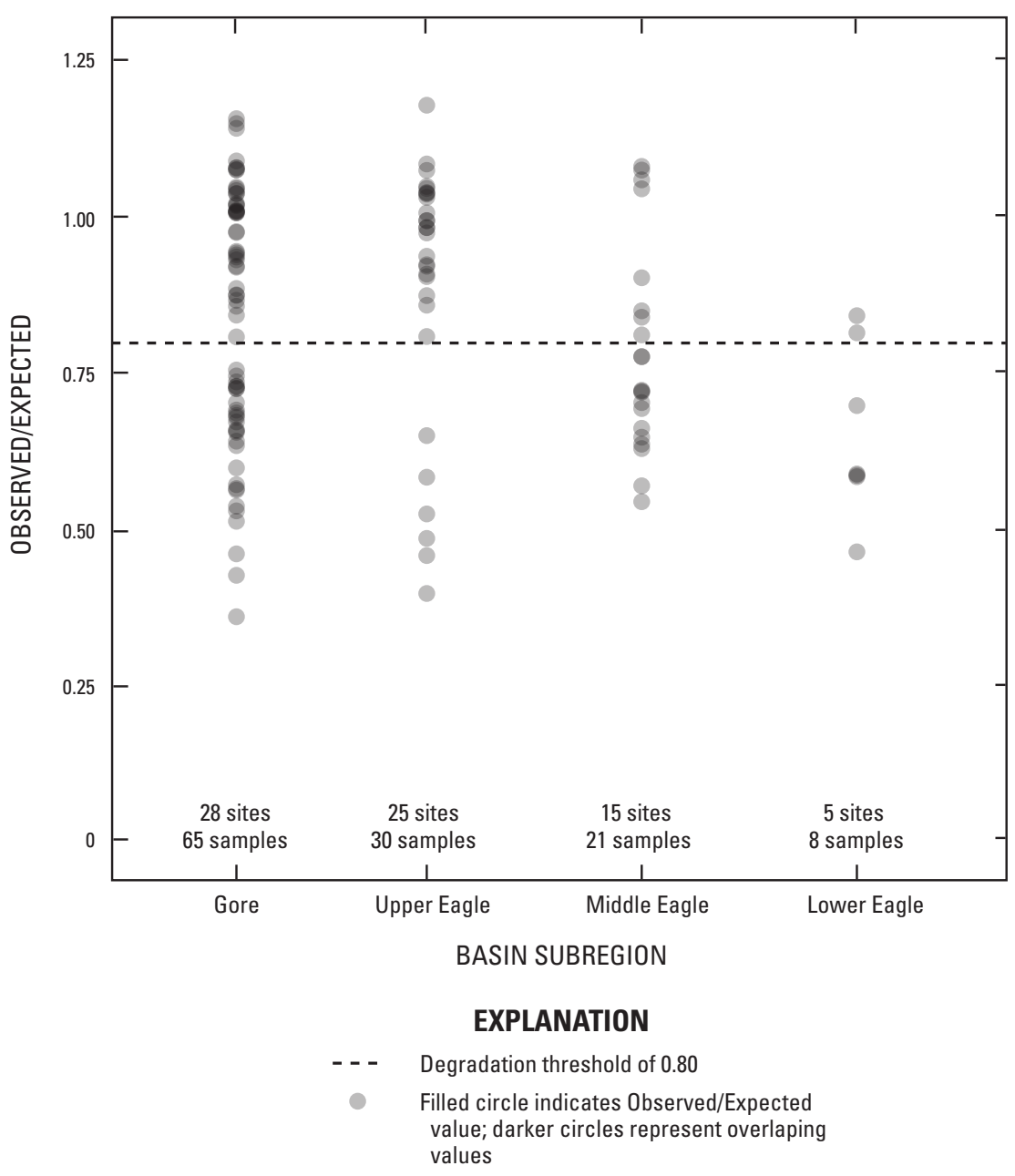

Figure 4. Summary of observed/expected values calculated from 124 macroinvertebrate samples collected at 73 sites grouped by different subregions of the Eagle River watershed, Colorado, 2000-07 (see text for subregion definitions).

Although most urban sites were considered biologically degraded, a few sites had $\mathrm{O} / \mathrm{E}$ values greater than the degraded condition threshold (sites 47, 57, 69, table 1). In a study of streams in the eastern United States, Carlisle and Meador (2007) observed that some urban sites with intact macroinvertebrate communities $(\mathrm{O} / \mathrm{E}>0.80)$ had well protected and established riparian corridors. In this study, there were no anecdotal explanations to describe why macroinvertebrate communities were relatively intact at a few urban sites. Unfortunately, the urban sites where $\mathrm{O} / \mathrm{E}$ was greater than 0.80 (sites $47,57,69$, table 1) have not been sampled since 2000-01, so results may not reflect current (2010) biological condition as urban development has increased since that time (2000-01) near these sites. If macroinvertebrate communities at these sites are intact under present-day (2010) land-cover conditions, then further investigation is warranted to better diagnose why these sites remain in good biological condition.
Mean $\mathrm{O} / \mathrm{E}$ for mixed sites was similar to that of urban sites; however, variation in $\mathrm{O} / \mathrm{E}$ values was largest when compared to other site classes (fig. 5). This result likely was affected by the inability to isolate the intensity of dominant factors or groups of co-occurring factors that affect biota at these mixed sites in the classification scheme used in this study. Others have suggested (for example, Carlisle and Hawkins, 2008) that variation in biological condition likely is associated with environmental factors operating at different spatial scales (that is, watershed, segment, and reach). Nonetheless, further investigation into causal factors affecting biological degradation at mixed sites will require better isolation of stressors through a well-planned study design. Understanding why some sites expected to have degraded biological communities (for example, urban and mixed sites), but show otherwise, will have clear implications for managing land use adjacent to streams and ultimately improving biological 


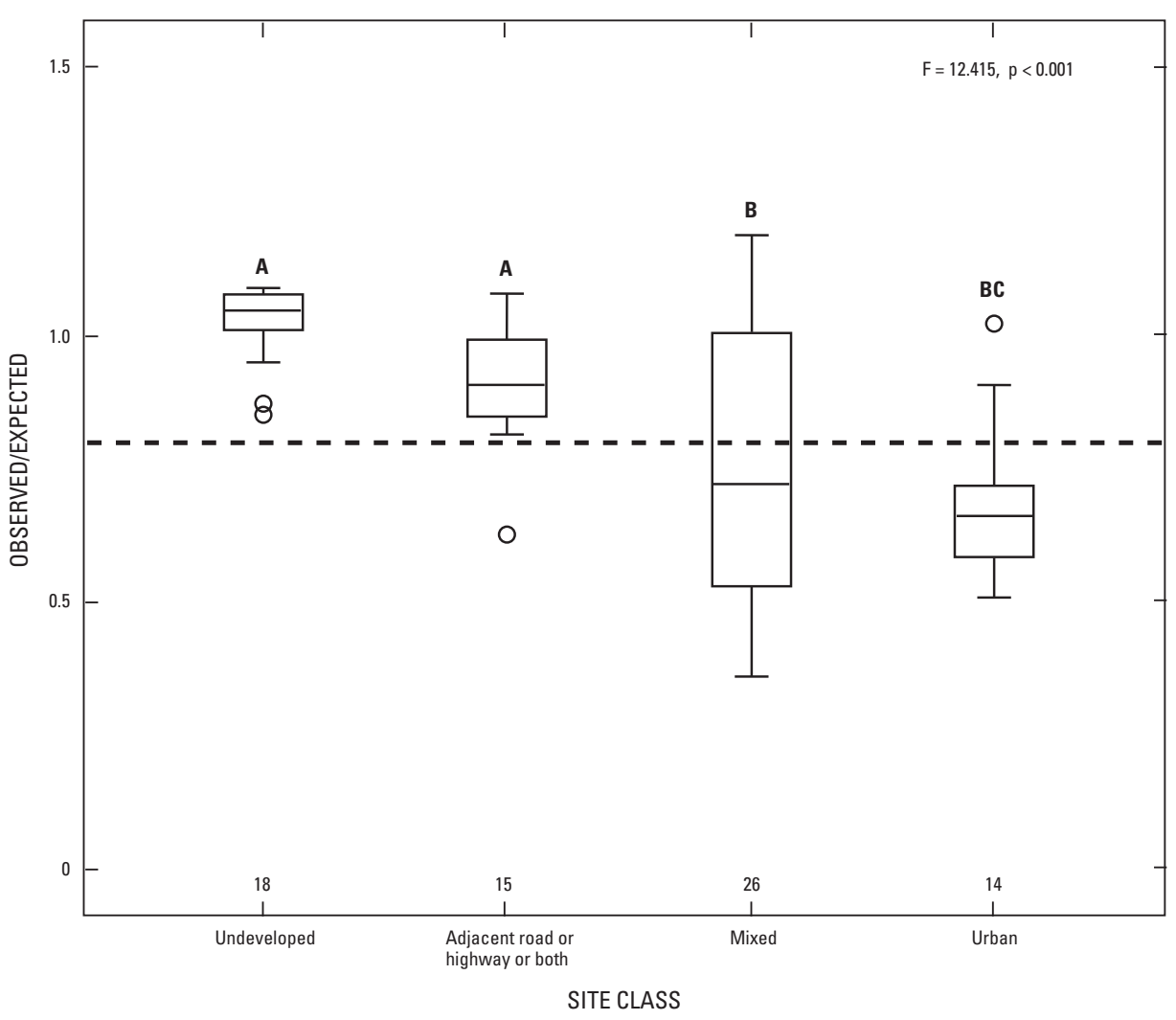

\section{EXPLANATION}

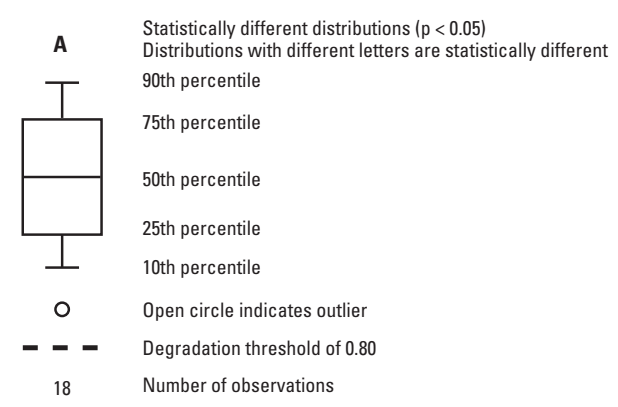

Figure 5. Distribution of macroinvertebrate observed/expected (O/E) values among undeveoped, adjacent road or highway or both, mixed, and urban site classes.

condition in the ERW at selected sites through mitigation efforts.

Mean O/E values from ARH sites were expected to be lower than undeveloped sites, but they were found to be indistinguishable (fig. 5). This result was somewhat surprising as many of the ARH sites previously were noted as being affected by sediment deposition associated with FS road networks (Laurie, 2003) or Colorado Department of Transportation's road sanding operations during the winter months (December through March) (for example, Black Gore Creek; Healy, 2008). Effects of sedimentation on macroinvertebrate communities are well documented (Waters, 1995 and citations within), causing significant reductions in macroinvertebrate density, biomass, and diversity (Lenat and others, 1981; Newcombe and MacDonald, 1991; Angradi, 1999). The results in this report likely were affected by the inability to quantify the intensity of sedimentation at each ARH site in the classification scheme used herein. Other factors potentially affecting macroinvertebrate community response include the degree to which adjacent roads were buffered from adjacent streams by riparian vegetation, or whether disturbed areas associated with roads were directly connected to streams (see Wemple and others, 1996); however, neither was quantified in this study. Additionally, O/E measures taxa loss which may not be 

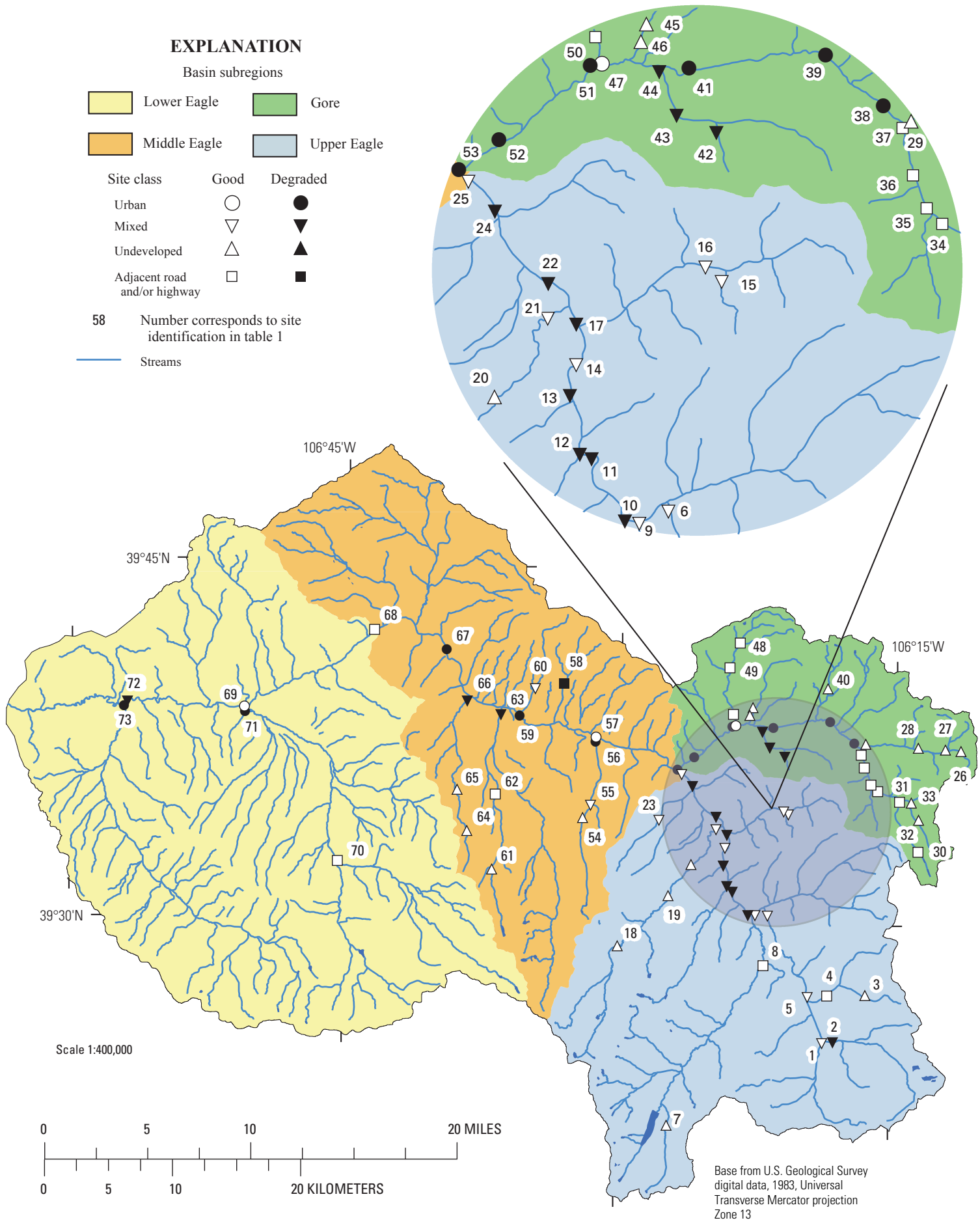

Figure 6. Site location, site class, biological condition, and Eagle River watershed subregions, Colorado. Biological condition was based on the most recent samples collected between 2000 and 2007. 
sensitive enough to indicate the intensity levels of sedimentation that differentially occurred across ARH sites. Furthermore, the most recent samples collected at each site were used in this analysis; and therefore, those samples may reflect sediment mitigation efforts implemented at some of these sites the year the samples were collected (for example, sites along Black Gore Creek) or may be a result of a decreased need to apply traction sand during that year (for example, a milder winter with less snow).

\section{Annual Variability of Observed/Expected}

Annual variability of $\mathrm{O} / \mathrm{E}$ values over a 3- to 5-year period visually differed between undeveloped sites and sites along Lower Gore and Black Gore Creeks (fig. 7). Assessments of biological condition at undeveloped sites consistently were above the 0.80 threshold (mean O/E, 1.01; Standard Deviation (SD), 0.07; Coefficient of Variation (CV), 6.85), indicating that these sites were consistently in good condition and stable over the sampling period; whereas, assessments made along Black Gore Creek (mean O/E, 0.85; SD, 0.017; $\mathrm{CV}, 20.67$ ) and lower Gore Creek sites (mean O/E, 0.67; SD, $0.14 ; \mathrm{CV}, 20.41$ ) were comparatively variable and year depedent (table 2).

At lower Gore Creek urban sites (table 1, sites 38, 39, 41, and 52), most of the annual variability (large CV values) can be attributed to differences between $\mathrm{O} / \mathrm{E}$ values from samples collected in $2000(\mathrm{O} / \mathrm{E}$ near 1.0) and those samples collected during 2004 to $2007(\mathrm{O} / \mathrm{E}<0.80)$. These results indicate that either sampling error (that is, error because of field sampling, laboratory processing, modeling, or natural variability) or some other unexplained sources of variability affected $\mathrm{O} / \mathrm{E}$ values, or major changes occurred between 2000 and 2004 along Gore Creek that severely altered macroinvertebrate community structure. Regardless of what affected $\mathrm{O} / \mathrm{E}$ values between 2000 and 2004 at sites along lower Gore Creek, all but one assessment made during 2004 and 2007 have indicated degraded biological condition as defined herein.

Relatively high annual variability also was observed at most Black Gore Creek sites (fig. 7, table 2). Black Gore Creek sites (table 1, sites 30, 31, 34, and 36) used in this analysis were located along the section of stream listed in 2002 as one of Colorado's impaired waters under the Clean Water Act of 1972 (303d) for sediment deposition (Healy, 2008). Although most $\mathrm{O} / \mathrm{E}$ values calculated from these sites were above the impairment threshold, several were below, and a few were among the lowest observed in this study. Potential factors influencing variability in biological condition among years at these sites is possibly associated with interacting annual differences among the amount of traction sand and deicing chemical applied, timing and maintenance of sediment retention basins, sediment control or stabilization projects, or differences in rainfall, snowmelt, and associated stream hydrology. Identifying the sources of variability affecting assessments of biological condition was beyond the scope of this study; however, understanding assessment variability is imperative to mitigation efforts (for example, sediment control efforts currently (2010) being lead by CDOT that began in 2002 (Healy, 2008) so that the uncertainty affecting any future mitigation efforts are minimized.

Table 2. Site and data description where 3 to 5 years of annual macroinvertebrate samples were collected in the Eagle River watershed, Colorado, 2000-07.

[ID, identification; $\mathrm{O} / \mathrm{E}$, ratio of observed to expected macroinvertebrate taxa, $>0.80$, biologically good, $<0.80$ biologically degraded; $>$, greater than; $<$, less than; CV, coefficient of variation]

\begin{tabular}{|c|c|c|c|c|c|c|}
\hline $\begin{array}{l}\text { Site ID } \\
\text { (fig. 2) }\end{array}$ & Stream & $\begin{array}{c}\text { Years of } \\
\text { data }\end{array}$ & $\begin{array}{l}\text { Site } \\
\text { class }\end{array}$ & $0 / E$ & $\begin{array}{c}\text { Mean } \\
0 / E\end{array}$ & $\begin{array}{c}\text { CV of } \\
0 / E\end{array}$ \\
\hline 18 & Cross Creek & 3 & Undeveloped & $>0.80$ & 1.04 & 4.03 \\
\hline 27 & Gore Creek & 3 & Undeveloped & $>0.80$ & 1.14 & 3.46 \\
\hline 28 & Gore Creek & 3 & Undeveloped & $>0.80$ & 0.95 & 7.69 \\
\hline 29 & Gore Creek & 5 & Undeveloped & $>0.80$ & 1.01 & 8.76 \\
\hline 32 & Polk Creek & 3 & Undeveloped & $>0.80$ & 0.94 & 10.66 \\
\hline 33 & Polk Creek & 3 & Undeveloped & $>0.80$ & 1.00 & 6.49 \\
\hline 30 & Black Gore Creek & 4 & Adjacent road or highway, or both & Year dependent & 0.70 & 26.72 \\
\hline 31 & Black Gore Creek & 4 & Adjacent road or highway, or both & Year dependent & 0.86 & 13.39 \\
\hline 34 & Black Gore Creek & 5 & Adjacent road or highway, or both & Year dependent & 0.87 & 35.36 \\
\hline 36 & Black Gore Creek & 4 & Adjacent road or highway, or both & $>0.80$ & 0.96 & 7.23 \\
\hline 38 & Gore Creek & 3 & Urban & $<0.80$ & 0.61 & 13.32 \\
\hline 39 & Gore Creek & 5 & Urban & Year dependent & 0.76 & 25.69 \\
\hline 41 & Gore Creek & 5 & Urban & Year dependent & 0.71 & 26.73 \\
\hline 52 & Gore Creek & 5 & Urban & $<0.80$ & 0.60 & 15.90 \\
\hline
\end{tabular}




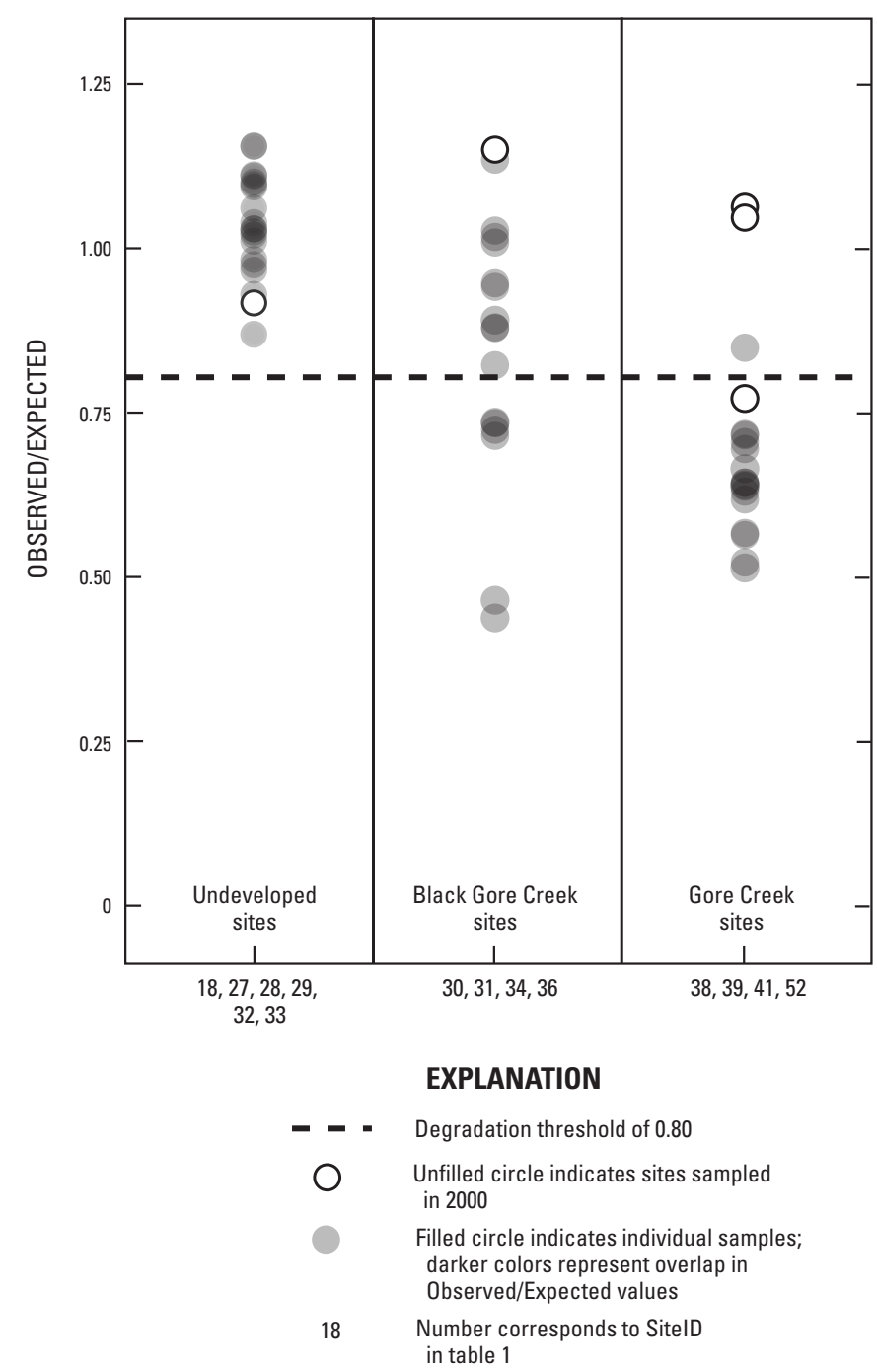

Figure 7. Distribution of observed/expected values collected from 14 sites ( 6 undeveloped sites, 20 samples; 4 Black Gore Creek sites, 17 samples; 4 Gore Creek sites, 18 samples) representing 3 to 5 years of annual variability between 2000 and 2007.

\section{Observed and Expected Macroinvertebrate Taxa at Urban Sites}

At urban sites, the occurrence of many macroinvertebrate taxa statistically was different than expected (fig. 8). Of the 173 taxa evaluated, 21 were observed or predicted to occur at 7 or more sites (that is, one-half of the total number of urban sites). Of these 21 taxa, 9 were observed less frequently (intolerant decreasers) and 4 were observed more frequently (tolerant increasers) than expected. Additionally, there were six taxa apparently tolerant (nonresponsive) to the conditions at urban sites as they were observed nearly as frequently as predicted. Combined, these 19 taxa provide an opportunity to enhance the interpretation of future studies in the ERW but will require better insight into the responses of these taxa to specific stressors.

In general, the patterns of tolerant increaser (hereafter increaser) and intolerant decreaser (hereafter decreaser) taxa are consistent with the findings of other studies. Many of the decreaser taxa observed belong to groups known to be sensitive (for example, mayflies, stoneflies, and caddisflies), and many of the increasers belong to groups known to be tolerant (for example, Oligochaeta, Turbellaria, Chironomidae) to general disturbance (Barbour and others, 1999). It is emphasized that these results are specific to this study for a variety of reasons (for example, small sample size, taxonomic resolution, and small geographic scale) and should not be applied outside the ERW until they are further studied. Nonetheless, the list of decreaser taxa (fig. 8) provides a starting point for identifying causal factors at urban sites responsible for degraded biological condition if further effort is made to better understand the ecological requirements of these taxa and their tolerance to more specific stressors. Integrating results of this study with other approaches such as field and laboratory experiments greatly will enhance abilities to identify causal factors associated with degraded biological condition (Clements and others, 2002). Information generated from such integrative studies will be imperative for well targeted mitigation efforts at urban sites in the ERW.

\section{Summary and Conclusions}

Local entities in the Eagle River watershed (ERW) are interested in better understanding how specific land-management activities affect biological communities and water quality in order to guide watershed-wide water-resource use management. In response to these needs, the U.S. Geological Survey (USGS), in cooperation with the Colorado River Water Conservation District, Eagle County, Eagle River Water and Sanitation District, Upper Eagle Regional Water Authority, Colorado Department of Transportation, City of Aurora, Town of Eagle, Town of Gypsum, Town of Minturn, Town of Vail, Vail Resorts, Colorado Springs Utilities, Denver Water, and the Department of U.S. Agriculture Forest Service (FS), compiled previously collected macroinvertebrate data in the ERW from selected USGS and FS studies from 2000-07. Specific objectives were to: (1) assess the biological condition of selected sites in the ERW using macroinvertebrate communities and (2) evaluate whether the dominant types of land cover were associated with biological condition. Additionally, short-term annual variability (3 to 5 years) in $\mathrm{O} / \mathrm{E}$ values was described and macroinvertebrate taxa that occurred more or less frequently than predicted at urban sites were identified.

Sites were classified into one of four classes (undeveloped, adjacent road or highway or both, mixed, and urban) using a combination of riparian land-cover characteristics, examination of topographic maps and aerial imagery, 


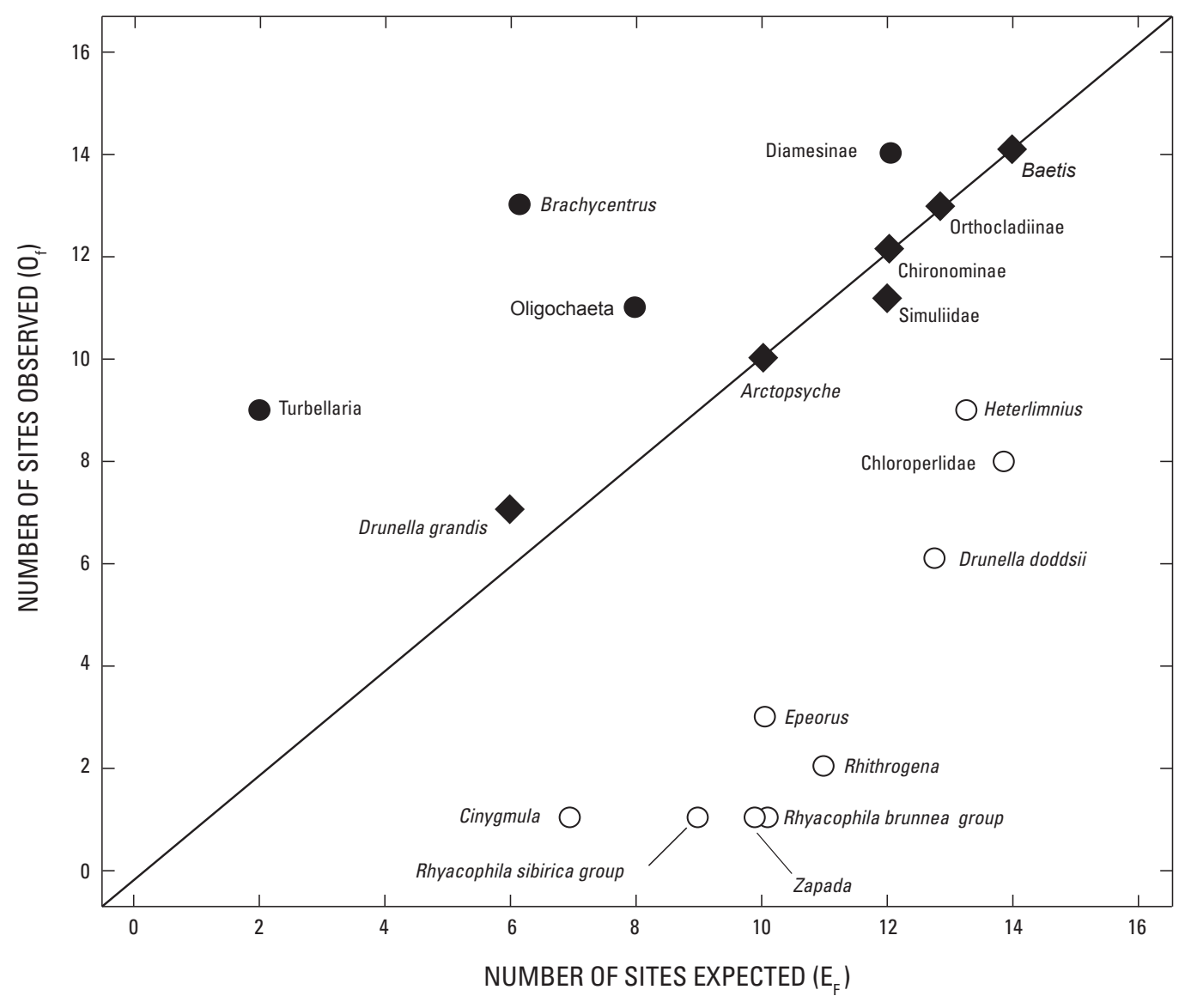

EXPLANATION

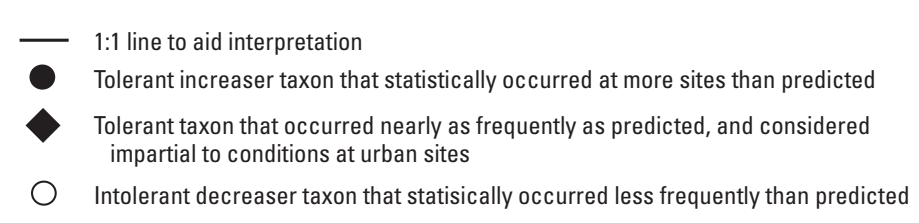

Figure 8. Frequencies of observed $\left(\mathrm{O}_{\mathrm{f}}\right)$ and expected $\left(\mathrm{E}_{\mathrm{f}}\right)$ occcurrence of 19 invertebrate taxa that statistically occurred more or less frequently than predicted or were impartial to conditions at urban sites in the Eagle River watershed, Colorado, 2000-07.

screening for exceedances in water-quality standards, and best professional judgment.

An independently developed predictive model was used to calculate a measure of taxonomic completeness for 124 macroinvertebrate samples collected from 73 sites in the ERW, where taxonomic completeness was expressed as the ratio of the number of observed $(\mathrm{O})$ taxa collected to those expected (E) to occur at each site in the absence of environmental degradation. These data were analyzed to assess the biological condition (that is, biologically "degraded" or "good") of selected sites. Analysis of variance was used to determine if site class accounted for variability in mean macroinvertebrate $\mathrm{O} / \mathrm{E}$ values. Macroinvertebrate communities were considered degraded at sites where $\mathrm{O} / \mathrm{E}$ values were less than 0.80 , indicating that at least 20 percent of expected taxa were not observed. This threshold also was statistically based as 0.80 was near the 10th percentile O/E value (0.79) of the distribution of reference site data used to develop the model. Also, macroinvertebrate taxa that increased (tolerant increasers), decreased (intolerant decreasers), or were nonresponsive to conditions at urban sites were identified. It is emphasized that these assessment results are specific to the models used herein and are not directly comparable to assessments made with different indicators, methods, models, or thresholds.

This study represents the first standardized assessment of biological condition of selected sites distributed across the ERW. Of the 73 sites evaluated, just over one-half (55 percent) were considered in good biological condition $(\mathrm{O} / \mathrm{E}$ 
greater than 0.80 ). The remaining sites either were biologically degraded (30 percent; $\mathrm{O} / \mathrm{E}$ less than 0.80 ) or conditions were dependent on the year sampled (15 percent; $\mathrm{O} / \mathrm{E}$ less than or greater than 0.80 depending on the year sampled). These assessments may not reflect current (2010) biological condition because 35 percent ( 26 sites) of these sites have not been sampled since 2000 or 2001 . Nonetheless, site assessments are considered a screening tool for determining where in the ERW biological conditions are good or degraded until further investigation confirms otherwise.

Sites primarily affected by urbanization were among the most severely degraded (urban sites had the lowest mean O/E values) when compared to other site classes. Although most urban sites were among the most severely degraded (that is, lowest mean $\mathrm{O} / \mathrm{E}$ values relative to other site classes), a few sites had nearly intact macroinvertebrate communities $(\mathrm{O} / \mathrm{E}$ near 1.0). Similar observations were noted among mixed classified sites. At least some within-class variability was possibly associated with errors inherent to classifying sites (that is, the inability to equally isolate dominate stressors at each site), especially for sites in the mixed class. Nonetheless, understanding why some sites were expected to have degraded biological communities, but showed otherwise, will have clear implications for mitigating biologically degraded streams in the ERB and elsewhere.

Apparent changes occurred in biological condition (that is, from good to degraded) between 2000 and 2004 at urban sites along Gore Creek because nearly all annual samples collected during 2004 to 2007 have indicated degraded conditions. At undeveloped sites, biological condition remained stable during 2000-05, whereas conditions were year dependent at some adjacent road or highway or both (ARH) sites along Black Gore Creek. Understanding the associated causes of annual variability in macroinvertebrate communities among Black Gore Creek sites, as well as apparent biological degradation along Gore Creek, is warranted to effectively target mitigation efforts in the Gore Creek watershed.

Thirteen macroinvertebrate taxa were identified that occurred more or less frequently than expected (that is, 4 taxa were tolerant increasers and 9 were intolerant decreasers) at urban sites. Additionally, six other taxa were impartial (tolerant) to the same conditions (that is, they occurred nearly as often as predicted). Combined, these 19 taxa provide an opportunity to enhance the interpretation of future studies in the ERW but will require better insight into the responses of these taxa to specific stressors. As a starting point, developing a better understanding of the ecological requirements and tolerance to specific stressors of the 9 decreaser taxa can enhance the ability to identify causal factors associated with degraded biological condition, ultimately directing mitigation efforts at urban sites in the ERW.

In conclusion, these results represent the first standardized assessment of biological condition of selected sites in the
ERW. More than one-half of the sites sampled were in good biological condition; however, nearly one-third of the sites sampled were considered biologically degraded. In most cases, sites in good biological condition were associated with undeveloped parts of the watershed (higher elevations); whereas, sites primarily affected by urbanization were among the most severely degraded (that is, lowest mean $\mathrm{O} / \mathrm{E}$ values when compared to other site classes). Understanding the sources of variability affecting biological assessments along with why some sites expected to be degraded, but showed otherwise, will have clear implications for mitigation efforts. Integrating results of this study with other approaches such as field and laboratory experiments will enhance abilities to identify causal factors associated with degraded biological condition. Information generated from such integrative studies will be imperative for well targeted mitigation efforts in the ERW.

\section{Acknowledgments}

The authors thank the Colorado River Water Conservation District, Eagle County, Eagle River Water and Sanitation District, Upper Eagle Regional Water Authority, Colorado Department of Transportation, City of Aurora, Town of Eagle, Town of Gypsum, Town of Minturn, Town of Vail, Vail Resorts, Colorado Springs Utilities, and Denver Water for their continued support of this program. The authors also thank all U.S. Geological Survey (USGS), U.S. Department of Agriculture Forest Service employees, and others who assisted in collecting field data evaluated in this study. Daren Carlisle (USGS Reston, Va.) and several other reviewers provided useful comments that greatly improved earlier versions of this manuscript.

\section{References Cited}

Angradi, T.R., 1999, Fine sediment and macroinvertebrate assemblages in Appalachian streams - a field experiment with biomonitoring applications: Journal of the North American Benthological Society, v. 18, p. 49-66.

Barbour, M.T., Gerritsen, J., Snyder, B.D., and Stribling, J.B., 1999, Rapid bioassessment protocols for use in streams and rivers - periphyton, benthic macroinvertebrates, and fish (2d ed.): Washington, D.C., U.S. Environmental Protection Agency, Office of Water, EPA-841-B-99-002, variously paged.

Bledsoe, B., Meyer, J., Holburn, E., Cuhaciyan, C., Earsom, S., Olson, K., and Snyder, B., 2005, Eagle River inventory and assessment: Avon, Colo., Eagle River watershed Council, 319 p. 
Cao, Y., Hawkins, C.P., and Storey, A.W., 2005, A method for measuring the comparability of different sampling methods used in biological surveys - implications for data integration and synthesis: Freshwater Biology, v. 50, p. 1, 105-1115.

Carlisle, D.M., and Hawkins, C.P., 2008, Land use and the structure of Western U.S. stream macroinvertebrate assemblages - predictive models and ecological traits: Journal of the North American Benthological Society, v. 27, p. 986-999.

Carlisle, D.M., Hawkins, C.P., Meador, M.R., Potapova, M., and Falcone, J., 2008, Biological assessments of Appalachian streams based on predictive models for fish, macroinvertebrate, and diatom assemblages: Journal of the North American Benthological Society, v. 27, p. 16-37.

Carlisle, D.M., and Meador, M.R., 2007, A biological assessment of streams in the Eastern United States using a predictive model for macroinvertebrate assemblages: Journal of the American Water Resources Association, v. 43, p. 1194-1207.

Clarke, R.T., Wright, J.F., and Furse, M.T., 2003, RIVPACS models for predicting the expected macroinvertebrate fauna and assessing the ecological quality of rivers: Ecological Modeling, v. 160, p. 219-233.

Clements, W.H., Carlisle, D.M., Courtney, L.A., and Harrahy, E.A., 2002, Integrating observational and experimental approaches to demonstrate causation in stream biomonitoring studies: Environmental Toxicology and Chemistry, v. 21 , p. 1138-1146.

Colorado Department of Local Government, 2003, Colorado preliminary population forecasts, forecast program methodology: Colorado Department of Local Affairs (DOLA), accessed June 2004, at http://dola.colorado.gov/demog/ PopulationTotals.cfm.

Cuffney, T.F., Gurtz, M.E., and Meador, M.R., 1993, Methods for collecting benthic macroinvertebrate samples as part of the National Water-Quality Assessment Program: U.S. Geological Survey Open-File Report 93-406, 40 p.

Davies, S.P., and Jackson, S.K., 2006, The biological condition gradient - a conceptual model for interpreting detrimental change in aquatic ecosystems: Ecological Applications, v. 16 , p. 1251-1266.

Deacon, J.R., and Spahr, N.E., 1998, Water-quality and biological community characterization at selected sites on the Eagle River, Colorado, September 1997 and February 1998: U.S. Geological Survey Water-Resources Investigations Report 98-4236, 8 p.
Fitzpatrick, F.A., Waite, I.R., D’Arconte, P.J., Meador, M.R., Maupin, M.A., and Gurtz, M.E., 1998, Revised methods for characterizing stream habitat in the National Water-Quality Assessment Program: U.S. Geological Survey WaterResource Investigations Report 98-4052, 67 p.

Hawkins, C.P., 2006, Taxonomic completeness as a measure of biological integrity: Ecological Applications, v. 16, p. $1277-1294$.

Hawkins, C.P., and Carlisle, D.M., 2001, Use of predictive models for assessing the biological integrity of wetlands and other aquatic habitats, in Rader, R., Batzer, D., and Wissinger, S., eds., Bioassessment and management of North American freshwater wetlands: New York, Wiley, p. 59-83.

Hawkins, C.P., Norris, R.H., Hogue, J.N., and Feminella, J.W., 2000, Development and evaluation of predictive models for measuring the biological integrity of streams: Ecological Applications, v. 10, p. 1456-1477.

Hawkins, C., Ostermiller, J., Vinson, M., and Stevenson, R.J., 2001, Stream algae, macroinvertebrate, and environmental sampling associated with biological water quality assessments field protocols: Logan, Utah State University, Department of Fisheries and Wildlife, Bureau of Land Management Aquatic Monitoring Center, 23 p.

Healy, Brian, 2008, Aquatic habitat and macroinvertebrate monitoring - Gore and Black Gore Creeks 2004-2007: Avon, Colo., Eagle River watershed Council, 16 p.

Hemsley-Flint, B., 2000, Classification of the biological quality of rivers in England and Wales, in Wright, J.F., Sutcliffe, D.W., and Furse, M.T., eds., Assessing the biological quality of fresh waters - RIVPACS and other techniques: Ambleside, Cumbria, United Kingdom, Freshwater Biological Association, p. 55-70.

Hydrosphere, 2007, Gore Creek algae investigation: Avon, Colo., Eagle River Water and Sanitation District, 23 p.

Klemm, D.J., Lewis, P.A., Fulk, F., Lazorchak, J.M., 1990, Macroinvertebrate field and laboratory methods for evaluating the biological integrity of surface waters: Cincinnati, Ohio, U.S. Environmental Protection Agency, Environmental Monitoring and Support Laboratory, EPA-600-4-90-030.

Laurie, G., 2003. Stream channel conditions in tributaries to Gore Creek and the Eagle River, Colorado: Silverthorne, Colorado, White River National Forest, 34 p.

Lenat, D.R., Penrose, D.L., and Eagleson, K.W., 1981, Variable effects of sediment addition on stream benthos: Hydrobiologia, v. 79, p. 187-194. 
Moss, D., Furse, M.T., Wright, J.F., and Armitage, P.D., 1987, The prediction of macroinvertebrate fauna of unpolluted running-water sites in Great Britain using environmental data: Freshwater Biology, v. 17, p. 41-52.

Multi-Resolution Land Characteristics Consortium, 2001, National land cover database for the conterminous United States: Multi-Resolution Land Characteristics Consortium, accessed October 15, 2008, at http://www.mrlc.gov/index. php.

National Oceanic and Atmospheric Administration, 2009, Parameter-elevation Regressions on Independent Slopes Model (PRISM) data, U.S. National Oceanic and Atmospheric Administration, National Climatic Data Center, accessed January 23, 2009, at http://www.ncdc.noaa.gov/oa/ ncdc.html.

Newcombe, C.P., and MacDonald, D.D., 1991, Effects of suspended sediments on aquatic ecosystems: North American Journal of Fisheries Management, v. 11, p. 72-82.

Ostermiller, J.D., and Hawkins, C.P., 2004, Effects of sampling error on bioassessments of stream ecosystemsapplication to RIVPACS-type models: Journal of the North American Benthological Society, v. 23, p. 363-382.

Paul, M.J., Gerritsen, J., Hawkins, C.P., and Leppo, E., 2005, Development of biological assessment tools for Colorado: Denver, Colorado Department of Public Health and Environment, Water Quality Control Division, Monitoring Unit, 58 p. (Also available at $h t t p: / / w w w . e p a . g o v /$ region8/ water/monitoring/FINAL\%20Colorado\%20DPHE\%20 Report_20051014.pdf.

Paul, M.J., and Meyer, J.L., 2001, Streams in the urban landscape: Annual Review of Ecology and Systematics, v. 32, p. 333-365.

Peterson, D.A., and Zumberge, J.R., 2006, Comparison of macroinvertebrate community structure between two riffle-based sampling protocols in Wyoming, Colorado, and Montana, 2000-2001: U.S. Geological Survey Scientific Investigations Report 2006-5117, 12 p.

Spatial Climate Analysis Service, 2004, Near-real time monthly high-resolution precipitation climate data set for the conterminous United States: accessed January 2009, at http://www.prism.oregonstate.edu/docs/meta/ppt_realtime_ monthly.htm.

SPSS, 2005, SPSS ver. 13.0 Brief guide, SPSS Inc.: Chicago, Illinois, $231 \mathrm{p}$.
Taylor, B.W., McIntosh, A.R., and Peckarsky, B.L., 2001, Sampling stream macroinvertebrates using electroshocking techniques - implications for basic and applied research: Canadian Journal of Fisheries and Aquatic Sciences, v. 58, p. 437-455.

Tweto, Ogden, 1979, The geologic map of Colorado: Special Publication, The Geologic Map of Colorado, U.S. Geological Survey, Reston, Virginia, scale 1:500,000 (reprinted).

U.S. Environmental Protection Agency, 2006, Wadeable streams assessment - a collaborative survey of the Nation's streams: Washington, D.C., U.S. Environmental Protection Agency, Office of Research and Development and Office of Water, EPA 841-B-06-002, 113 p. (Also available at http:// www.epa.gov/owow/streamsurvey/.)

U.S. Geological Survey, 2007, The National map seamless server: U.S. Geological Survey, accessed October 15, 2008, at $h t t p: / / s e a m l e s s . u s g s . g o v /$.

Van Sickle, J., Hawkins, C.P., Larsen, D.P., and Herlihy, A.T., 2005, A null model for the expected macroinvertebrate assemblages in streams: Journal of the North American Benthological Society, v. 24, p. 178-191.

Vieira, Nicole, Albeke, S., Rollings, A., 2005, Factors influencing brown trout population in mine-impacted reaches of the Eagle River following remediation efforts: Fort Collins, Colo., Colorado Division of Wildlife, 56 p.

Vinson, M.R., and Hawkins, C.P., 1996, Effects of sampling area and subsampling procedure on comparisons of taxa richness among streams: Journal of the North American Benthological Society, v. 15, p. 392-399.

Waters, T.F., 1995, Sediment in streams - sources, biological effects, and control: Bethesda, Md., American Fisheries Society, $251 \mathrm{p}$.

Wemple, B.C., Jones, J.A., and Grant, G.E., 1996, Channel network extension by logging roads in two basins, western Cascades, Oregon: Water Resources Bulletin, v. 32, p. 1195-1207.

Wilson, A.B., and Sims, P.K., 2003, Colorado mineral belt revisited - An analysis of new data: U.S. Geological Survey Open-File Report 03-046, 7 p.

Wynn, K.H., Bauch, N.J., and Driver, N.E., 2001, Gore Creek watershed, Colorado assessment of historical and current water-quantity, water quality, and aquatic ecology, 1968-98: U.S. Geological Survey Water-Resources Investigations Report 99-4270, $72 \mathrm{p}$.

Zuellig, R.E., and Bruce J.F., 2010, Macroinvertebrate and algal community sample collection methods and data collected at selected sites in the Eagle River watershed: U.S. Geological Survey Data Series 502, 81 p. 
Publishing support provided by:

Denver Publishing Service Center

For more information concerning this publication, contact:

Director, USGS Colorado Water Science Center

Box 25046, Mail Stop 415

Denver, CO 80225

(303) 236-4882

Or visit the Colorado Water Science Center Web site at:

http://co.water.usgs.gov/ 


\section{$\frac{2}{5}$}

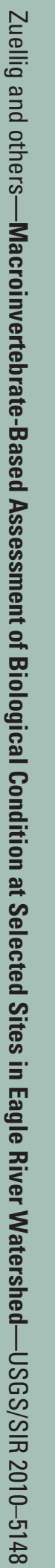

8 Printed on recycled paper

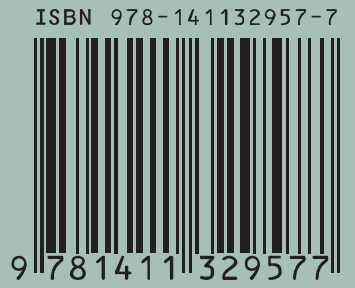

